\title{
A Comparative Study on Sideband Optimization in Time-Modulated Arrays
}

\author{
Ertugrul Aksoy and Erkan Afacan \\ Department of Electrical \& Electronics Engineering, Faculty of Engineering, Gazi University, 06570 Ankara, Turkey \\ Correspondence should be addressed to Ertugrul Aksoy; ertugrulaksoy@gazi.edu.tr
}

Received 29 November 2013; Accepted 19 March 2014; Published 29 April 2014

Academic Editor: Paolo Rocca

Copyright ( 2014 E. Aksoy and E. Afacan. This is an open access article distributed under the Creative Commons Attribution License, which permits unrestricted use, distribution, and reproduction in any medium, provided the original work is properly cited.

A comparative analysis of the three different methods known as sideband level suppression (SBL), sideband radiation suppression (SR), and sideband bound suppression (SB) which are used in sideband calculations for time-modulated arrays (TMAs) is presented. The three methods are investigated in terms of sideband level, sideband power, time average directivity, and solution time for TMAs so as to show the performances and potentialities of these distinct techniques. The problems of interference suppression, sidelobe cancelling, and joint interference suppression and sidelobe cancelling are analyzed for a time-modulated linear array. In the linear array 30 elements are used and the interelement spacing is determined to be a half-wavelength. During the investigations, differential evolution (DE) algorithm is chosen as the optimization tool. The common variable aperture size (VAS) time schemes are optimized to synthesize the desired goals in order to compare the techniques. The results show that the SB method is more convenient than the other two compared methods.

\section{Introduction}

The application of time as a fourth design parameter has been first suggested by Shanks and Bickmore in the late 1950s [1]. This first work has been followed by the work of Kummer et al. in 1963 in which time parameter is used to obtain an ultralow sidelobe pattern for a linear 8-element slot array [2]. After these works the time parameter has begun to be used for the improvement of the properties of antenna arrays [3]. The research in this area has gained acceleration especially with the advancement of calculation speed and with the emergence of new optimization techniques in the early 2000s. The first works were about obtaining the desired pattern at the operating frequency and controlling sidebands simultaneously [4-8] and also about investigating several switching sequences such as variable aperture size (VAS) [9], pulse shifting [10], and pulse splitting [11-14]. Also the time modulation implementation of several optimization methods such as differential evolution (DE) $[4,5]$, particle swarm optimization (PSO) [6], or simulated annealing (SA) [7] may be considered amongst the early studies. Time modulation has been also used in interference suppression and adaptive beamforming applications [15-18].

Sidebands occurring due to the periodic nature of time modulation are generally evaluated as a loss in many standard applications, but in the work of Tennant and Chambers, it is shown that these sidebands can be used on purpose [19]. Firstly, it is proposed that for a two-element time-modulated array a steerable deep null at the first harmonic frequency can be found and used in direction finding applications [19], and then this is proved experimentally in [20]. Later Li et al. have suggested that the main beam of the first harmonic can be steered without using phase shifters and can be used for communications [21]. Other direction finding applications using time modulation are given by $\mathrm{Li}$ et al. [22] and Hong et al. [23]. Recent studies about time modulation can be expressed as follows: gain improvement by using singlepole double-throw switches is investigated in [24]; reflector arrays with time modulation are investigated in [25]. The optimization of switching durations so as to maintain main beam directivity constant is given in [26]. Instead of switching functions with ideal rectangulars, more realistic modellings 
of pulses have been given in [27], and the effects on the radiation pattern have been also investigated.

The calculations towards sideband control were used to be realized by the sampling of a certain number of harmonic frequencies with a certain precision and by the usage of these samples as given in [4], since the number of sidebands is infinite. In 2008, the total radiated power for this infinite number of harmonics has been written as a closed-form finite summation by Brégains et al. [28]. With the emergence of this new method and by using the mentioned summation equation, it has been possible to calculate the total power at sidebands for linear arrays and under VAS switching. This equation has been generalized to planar arrays in $[29,30]$ and to pulse shift switching in [31]. Recently, it has been suggested by Aksoy and Afacan that the sideband control can be maintained by using an equation which limits the sidebands instead of using the sidebands themselves directly [32]. In this work, the three mentioned methods have been compared in terms of sideband level, sideband power, timeaverage directivity, and calculation time for three different problems.

The work is organized as follows. In Section 2 basic time modulation concept is summarized, in Section 3 the three mentioned sideband control methods are defined, in Section 4 the analysis of the results is given, and in Section 5 the results are discussed. The work is concluded in Section 6.

\section{Time Modulation}

In classical theory there exist three main design variables given as excitation amplitudes $(I)$, excitation phases $(\beta)$, and the position vectors that specify the array properties. Apart from the classical theory, it is asserted that the time-average radiated power may be adjusted by changing turn-on times of the array elements. By this way, the time parameter emerges as the fourth design parameter. The time modulation may be simply defined as the process of periodic switching of the array elements by high speed RF devices. For simplicity, consider a linear array consisting of isotropic sources whose elements are placed on the positive $z$-axis. The array factor (AF) for this array may be written as

$$
F(\theta)=\sum_{n=0}^{N-1} I_{n} e^{j \beta_{n}} e^{j k z_{n} \cos \theta}
$$

Here, $I_{n}, \beta_{n}$, and $z_{n}$ represent the $n$th element excitation amplitude, excitation phase, and the Euclidean distance to the system origin, respectively. Additionally $k=2 \pi / \lambda$ is the free space wave number and the angle $\theta$ is measured from the $z$-axis. If it is assumed that this architecture is switched by the variable aperture size (VAS) time scheme as shown in Figure 1, it is clear that a time-dependent variable will appear in the array factor.

If the switching function is modelled as

$$
U_{n}(t)= \begin{cases}1, & 0 \leq t \leq \tau_{n}^{\prime} \leq T_{p} \\ 0, & \text { otherwise }\end{cases}
$$

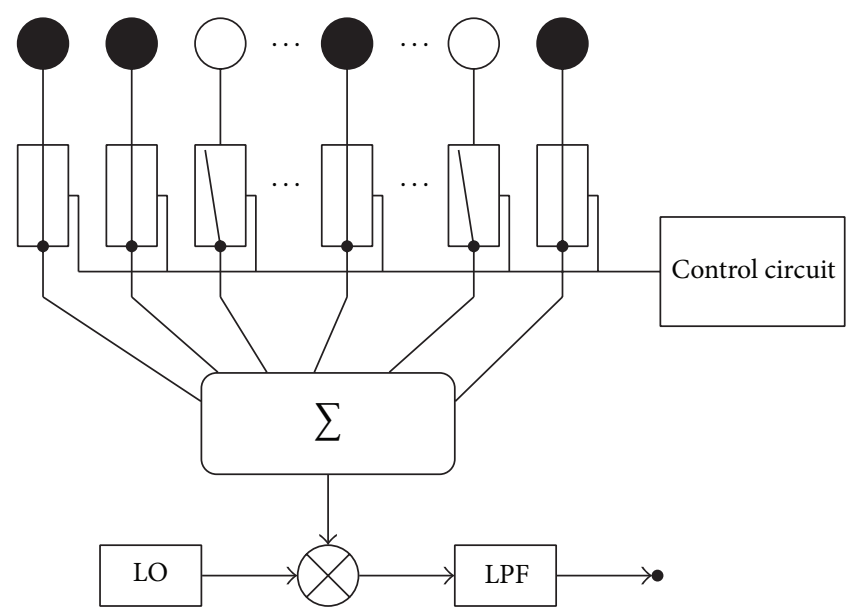

FIGURE 1: An illustration of a TM array receiver structure.

the time-dependent array factor becomes

$$
F(\theta, t)=e^{j \omega_{0} t} \sum_{n=0}^{N-1} I_{n} e^{j \beta_{n}} U_{n}(t) e^{j k z_{n} \cos \theta} .
$$

In this expression $U_{n}(t)$ is a periodic function in time domain and it is clear that it can be decomposed into complex Fourier series. Here it must be noted that there exist many different time schemes for time-modulated arrays (TMAs), but the common point is that they are periodic in time. If (2) is decomposed into Fourier series and substituted into its place, the time-dependent array factor will be

$$
F(\theta, t)=\sum_{m=-\infty}^{\infty} \sum_{n=0}^{N-1} I_{n} e^{j \beta_{n}} C_{n}^{m} e^{j k z_{n} \cos \theta} e^{j\left(\omega_{0}+m \omega_{p}\right) t} .
$$

Here, $C_{n}^{m}$ is the complex Fourier coefficient which belongs to $n$th element and $\omega_{0}$ and $\omega_{p}$ represent the angular carrier frequency and angular switching frequency, respectively. As it is understood from the equation, $m=0$ term represents the main operating band and other higher order terms are called the sidebands. For the VAS switching scheme the Fourier coefficient at central frequency may be written as $C_{n}^{0}=\tau_{n}^{\prime} / T_{p}=\tau_{n}$ and at $m$ th harmonic it may be written as $C_{n}^{m}=\tau_{n} \operatorname{sinc}\left(\pi m \tau_{n}\right) e^{-j \pi m \tau_{n}}$. Here $\operatorname{sinc}(x)$ is defined as $\operatorname{sinc}(x)=\sin x / x$. Therefore, when the switching frequency is much bigger than the carrier frequency and under the far field approximation, the array factor for a time-modulated array at the operating frequency can be written as

$$
F_{0}(\theta, t)=\sum_{n=0}^{N-1} I_{n} e^{j \beta_{n}} \tau_{n} e^{j k z_{n} \cos \theta},
$$

and for sidebands it may be written as

$$
F_{m}(\theta, t)=\sum_{n=0}^{N-1} I_{n} \tau_{n} \operatorname{sinc}\left(\pi m \tau_{n}\right) e^{j\left(k z_{n} \cos \theta-\pi m \tau_{n}+\beta_{n}\right)} .
$$


As it can be understood from these expressions, the time modulation inevitably shifts some of the radiated power into sidebands. Although these radiations may be used on purpose, in many standard applications they are considered as power losses and are put on trial to be suppressed. Since the sidebands are infinite in number, this process seems to be a hard problem and in the literature some methods are proposed to control the sidebands.

\section{Sideband Calculations}

For the problems in which sidebands are considered as waste of power, the sidebands require being suppressed in order to minimize the loss. The sideband suppression methods which are applicable to this type of problems may be categorized in three main groups as follows: (a) maximum sideband level suppression (SBL), (b) sideband radiation (SR) suppression, and a newly asserted idea (c) sideband bound (SB) suppression.

3.1. SBL Suppression. The first of the sideband calculation techniques is conducted by reducing the maximum radiation values for a specific number of harmonics as in sidelobe calculations. Although this technique is the first asserted one and may be applied to the cases where the maximum values of sidebands require being kept below a certain communication threshold, it needs a relatively long solution time depending on the problem at hand. As depicted in Figure 2 in detail, the technique may be defined as the process of sampling the desired number of sideband radiation patterns with a certain sensitivity and extracting the maximum level amongst these samples and then conducting a minimization procedure to this sampled and extracted level. Specifically, since the harmonic radiations are infinite in number, taking the first $N$ harmonics into account as conducted in early researches lengthens the solution time tremendously. As a remedy to this situation, it is assumed that the sidebands should decrease by a specific sinc function envelope, and that it should be sufficient to take the first harmonic into account instead of the first $N$ harmonics. This assumption is a partially true assumption depending on the problem, but it should be kept in mind that there exist situations in which the first harmonic level is not the highest. Additionally, it should be noted that, since the whole radiation space needs to be sampled, this technique requires tremendously long solution times whenever the problem does not have an azimuthal symmetry such as problems involving planar and volumetric geometries. For this type of problems a trade-off between accuracy-solution time or accuracy-system resources becomes inevitable.

3.2. SR Suppression. In 2008, Brégains et al. [28] have presented a closed-form finite summation which enables the calculation of the total radiated power at sidebands. SR technique is proposed by Poli et al. in 2010 [6] and it depends on the mentioned summation given in [28]. The original formulation given in [28] could be only used for specific conditions. After the reformulation by Poli et al. [29] and by Aksoy and Afacan [30,31], it has been possible to use the mentioned summation for a wider range of applications. Unlike SBL method, SR method mainly depends on the idea of suppressing the sidebands by minimizing the total radiated power at sidebands instead of minimizing the maximum sideband values. As depicted in Figure 3 in detail, the method works on the principle of direct reduction of

$$
\begin{aligned}
P_{\mathrm{SR}}= & 2 \pi \sum_{n}\left|I_{n}\right|^{2}\left[\tau_{n}\left(1-\tau_{n}\right)\right] \\
& +2 \pi \sum_{\substack{m, n=0 \\
m \neq n}}^{N-1} I_{n} I_{m}^{*}\left(\overline{\tau_{m n}}-\tau_{n} \tau_{m}\right) \operatorname{sinc}(R) .
\end{aligned}
$$

In this expression, $P_{\mathrm{SR}}$ represents the total power radiated at sidebands, $I_{n}$ represents the complex element excitations, $\tau_{n}$ and $\tau_{m}$ denote the $n$th and $m$ th elements normalized switch-on duration, respectively. $\overline{\tau_{m n}}$ represents the normalized overlapped duration of $m$ th and $n$th elements. $R$ is defined as $R=k\left(z_{m}-z_{n}\right)$ for linear arrays and $R=$ $k \sqrt{\left(x_{m}-x_{n}\right)^{2}+\left(y_{m}-y_{n}\right)^{2}}$ for planar arrays. This method provides faster solution time compared to SBL method for linear and planar arrays. It, also, ensures the maximization of the radiated power at central operating frequency since the lost power is directly minimized. However, one handicap of the technique is the absence of sideband level control term in (7). The method may produce undesired results for the problems in which the sideband level has to stay below a certain level [33].

3.3. SB Suppression. This method has been recently asserted by Aksoy and Afacan [32] to overcome the handicaps of SBL and SR techniques. Unlike SBL method, it works on the principle of minimizing a sideband bound which covers the infinitude of sidebands instead of reducing the maximum value points of first $N$ harmonics. Thereby, the long solution time which is the handicap of SBL minimization is shortened as well as the control of maximum sideband level point which is the handicap of SR technique is provided for both linear and planar geometries. Additionally, since this technique is a process of suppression of all harmonics at the same time, it ensures the control of the radiated power associated with sidebands. As shown in Figure 4, the method works on the principle of directly minimizing the normalized sideband bound which is given by

$$
\Psi=\left(\frac{\sum_{n=0}^{N-1} I_{n} \sin \left(\pi \tau_{n}\right)}{\pi \sum_{n=0}^{N-1} \xi_{n}}\right)^{2} .
$$

In this expression, $\Psi, I_{n}$, and $\tau_{n}$ represent the normalized upper sideband limit, the excitation amplitude of the related elements, and the normalized switch-on durations of related elements with respect to switching period, respectively. Also, $\xi_{n}$ is called the dynamic excitation amplitude defined by $\xi_{n}=I_{n} \tau_{n}$. 


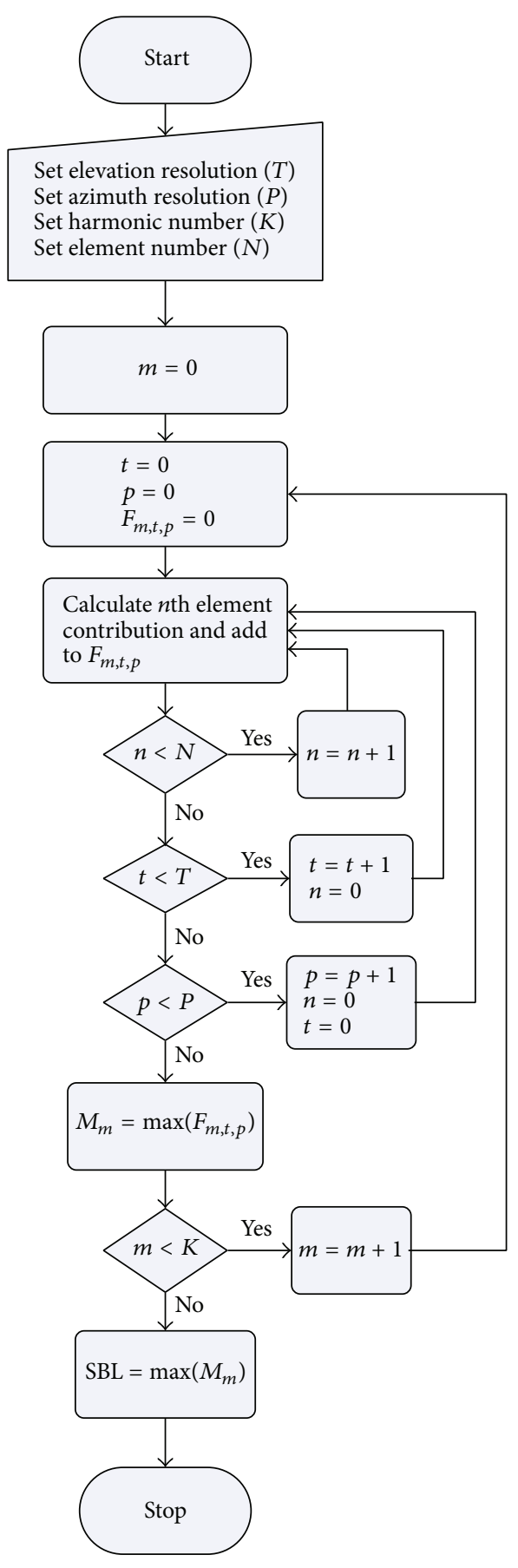

FIGURE 2: The illustrative flowchart of the SBL method.

\section{Analysis and Results}

In this section the three sideband suppression methods detailed in Section 3 have been compared for three different problems in terms of potentials and performances. The three problems are determined to be interference suppression, sidelobe cancelling, and joint interference suppression and sidelobe cancelling. A 30-element time-modulated linear array placed at the positive $z$-axis has been investigated.

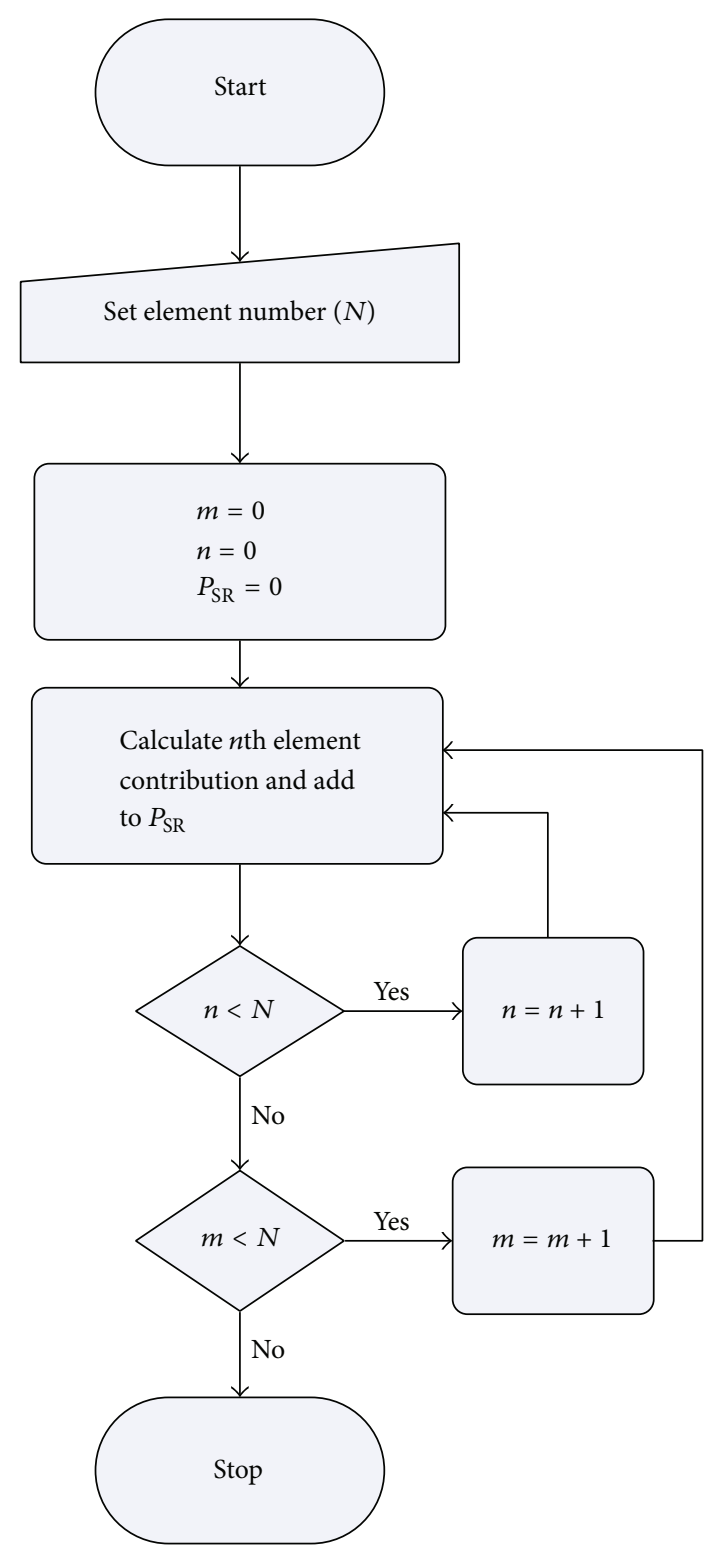

FIGURE 3: The illustrative flowchart of the SR method.

The array elements are assumed to be isotropic and the spacing between elements is accepted to be halfwavelength. Differential evolution algorithm with the scheme "DE/best/1/bin" is used as the optimization method. The population size, scaling factor, and crossover probability are selected as $P=20, F=0.63$, and $C_{r}=0.9$, respectively. In an optimization problem involving 30 parameters, usually 500 iterations are enough to obtain a converged result. However, in order to show that the results achieve steady values, the iteration limit is taken as 1000 . Hence, the termination condition of the algorithm is selected as 1000 iterations and 100 different independent experiments have been realized. For all of the analysis, a standard PC having $2 \mathrm{~GB}$ RAM and $2.01 \mathrm{GHz}$ CPU clock speed is used. For the analysis of the methods the interference suppression problem can be considered first. 


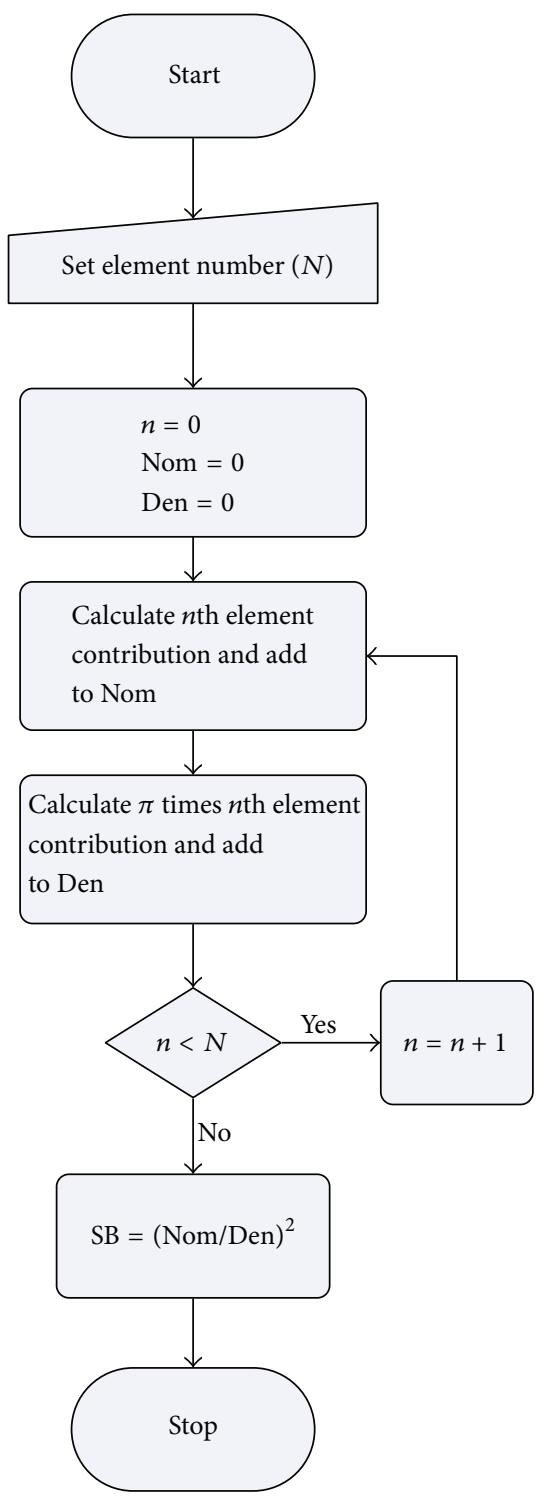

FIGURE 4: The illustrative flowchart of the SB method.

4.1. Interference Suppression (IS). In this problem the suppression at 30-degree elevation angle has been considered for the mentioned linear array and the cost function given by

$$
\Psi^{k}=\Upsilon^{k}+\Lambda^{k}
$$

is minimized to optimize switch-on times by using VAS switching scheme only. In (9) $\Upsilon$ represents the radiation value at the elevation angle which is desired to be suppressed, and $\Lambda$ represents the value obtained from the method used for sideband control. The upper index $k$ shows the method used (i.e., $k=1,2,3$ is used for SBL, SR, and SB, resp.).

The switch-on times and radiation patterns for the solutions having best convergence ratio amongst 100 experiments are shown in Figures 5 and 6, respectively. As is shown in Figure 6 , all three methods have maintained a sideband level below $-50 \mathrm{~dB}$. Interference suppression has been realized in the main radiation beam, but patterns have high sidelobe

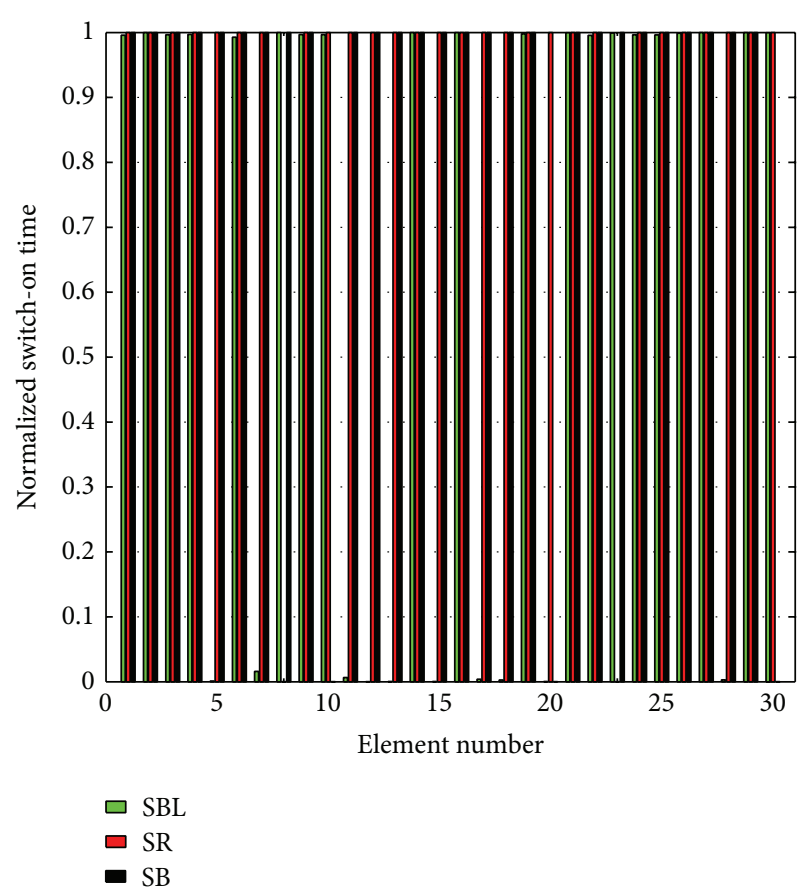

FIGURE 5: Switch-on time durations of the best converged solution for IS.

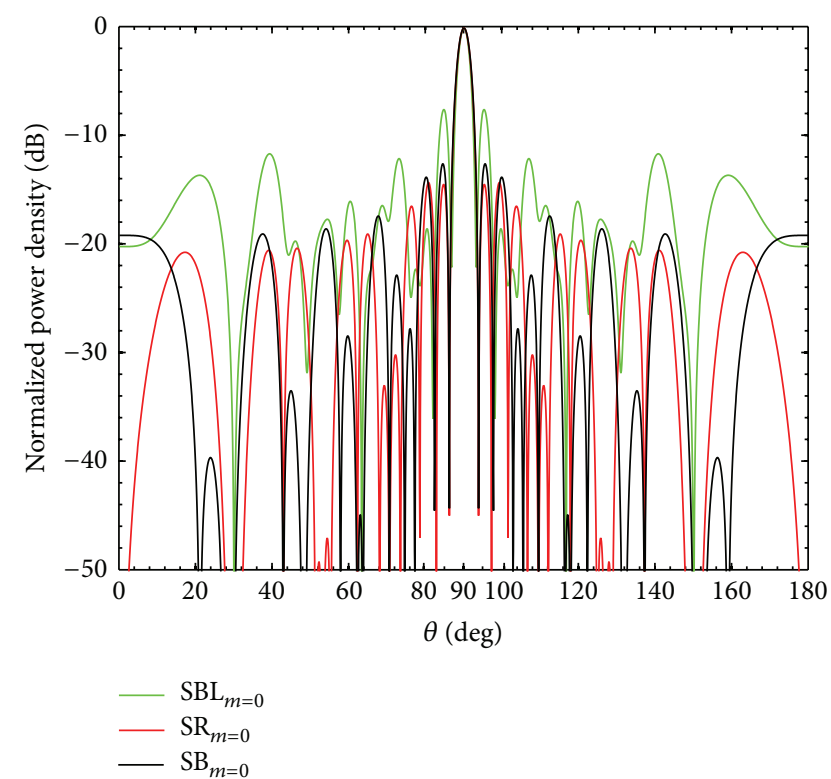

FIGURE 6: Radiation patterns at carrier frequency and at first harmonic related to Figure 5 for IS-the $m$ subscript represents the harmonic number. (Since all sidebands are below $-50 \mathrm{~dB}$ (see Figure 7), they are not shown in this figure.)

levels since sidelobe level is not taken into account in the problem. Specifically, sideband levels for the first 30 harmonics are given in Figure 7. As is seen from Figure 7, SR and SB methods have reduced all harmonics below $-130 \mathrm{~dB}$ for the best solution, but for the SBL method sideband level has been trapped in the $(-50)-(-60) \mathrm{dB}$ band. 
TABLE 1: Calculated benchmark parameters of DE algorithm outputs for IS.

\begin{tabular}{|c|c|c|c|c|c|c|c|c|c|}
\hline & & & & & & & & & \\
\hline & & SBL & & & SR & & & SB & \\
\hline & Best & Worst & Mean & Best & Worst & Mean & Best & Worst & Mean \\
\hline$\Psi$ & 0.002 & 0.040 & 0.019 & 0.000 & 0.036 & 0.014 & 0.000 & 0.040 & 0.015 \\
\hline$\Omega(\mathrm{dB})$ & -56.471 & -28.039 & -35.852 & -141.235 & -26.984 & -52.229 & -135.630 & -27.923 & -78.799 \\
\hline$P(\%)$ & 0.372 & 7.921 & 3.939 & 0.000 & 3.556 & 1.264 & 0.000 & 3.165 & 0.755 \\
\hline$D(\mathrm{~dB})$ & 13.017 & 12.498 & 13.074 & 14.472 & 13.061 & 13.838 & 14.314 & 13.696 & 13.854 \\
\hline
\end{tabular}

$\Psi$ represents the cost function value, $\Omega$ represents the maximum sideband level, $P$ represents the power loss percentage, and $D$ represents the time-average directivity.

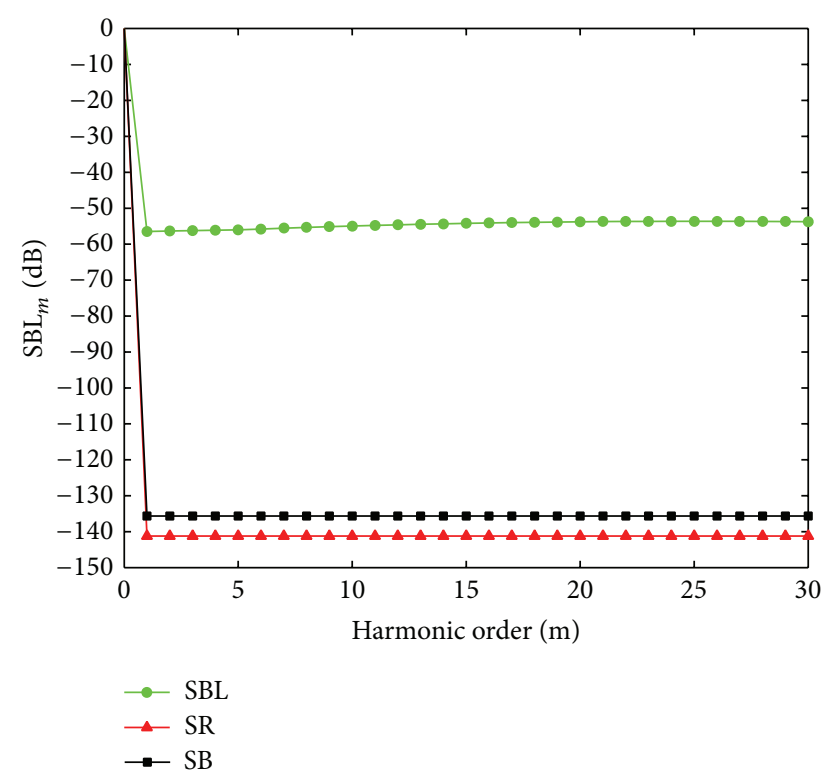

FIGURE 7: The first 30 maximum harmonic levels of best converged solution related to Figure 5 for IS.

The best, the worst, and the average values are tabulated in Table 1 and for each of the three methods the average convergence behaviour observed during the 100 experiments is presented in Figure 8. Referring to first row of Table 1, the best solutions have converged to close values for the three methods. Whenever the average convergence curves are examined, it can be seen from Figure 8 that although SBL method has a steeper descent at the beginning; finally it is behind SR and SB methods which converge closely.

Since the methods are used for the suppression of sidebands, the variation of sidebands during the optimization process can be observed. The mean maximum sideband leveliteration graphics is presented in Figure 9. As it is understood from the second row of Table 1, these three methods which show a similar behaviour for the worst solutions have been insufficient for sideband level suppression for the worst-case scenario of this problem. This insufficiency is evaluated to be a result of the random based operator characteristics of the optimization algorithm used. Whenever the best solutions are considered, sideband suppression ratios for SR and SB methods are close to each other and ahead of SBL technique.

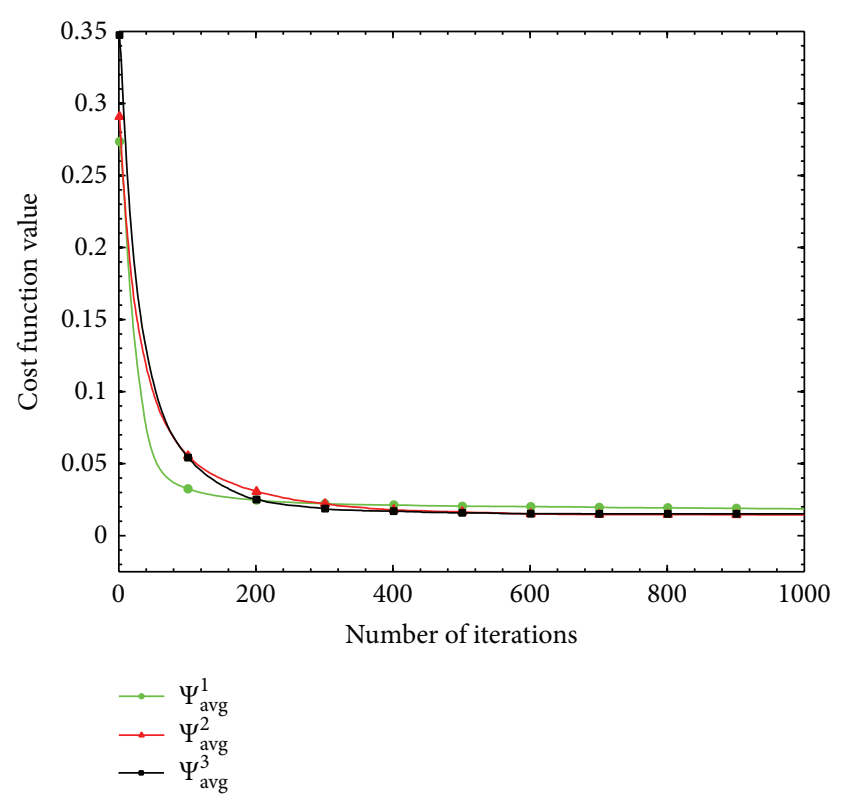

FIGURE 8: The average convergence behaviour of the three methods for IS $-\Psi^{k}$ represents the cost function value and $k$ represents the method used, where $k=1$ : SBL, $k=2: \mathrm{SR}$, and $k=3$ : SB.

For average values, as it can be seen from Figure 9, SB method has been more successful with respect to other two methods in suppressing the sidebands. Moreover, while in early iterations the SBL method exhibits a rapid suppression tendency compared to SB and SR methods, in average it falls behind the SR and SB techniques and if the average slopes of SB and SR methods are considered, it can be said that in every iteration SB method exhibits more efficient suppression compared to SR.

One of the advantages of SR method is the direct suppression of sideband powers. So, the variation of sideband power during the execution time of the algorithm can be investigated. For this case, sideband power loss in terms of percent versus iteration graphics is presented in Figure 10 and the results obtained from the best and the worst solutions are given in the third row of Table 1 . Regarding the average values, the sideband power loss is calculated to be close to $\% 4, \% 1.27$, and \%0.76 for SBL, SR, and SB methods, respectively, all for 1000 iterations, and also it is remarkable that SB method is the method which reduces sideband power fastest. 


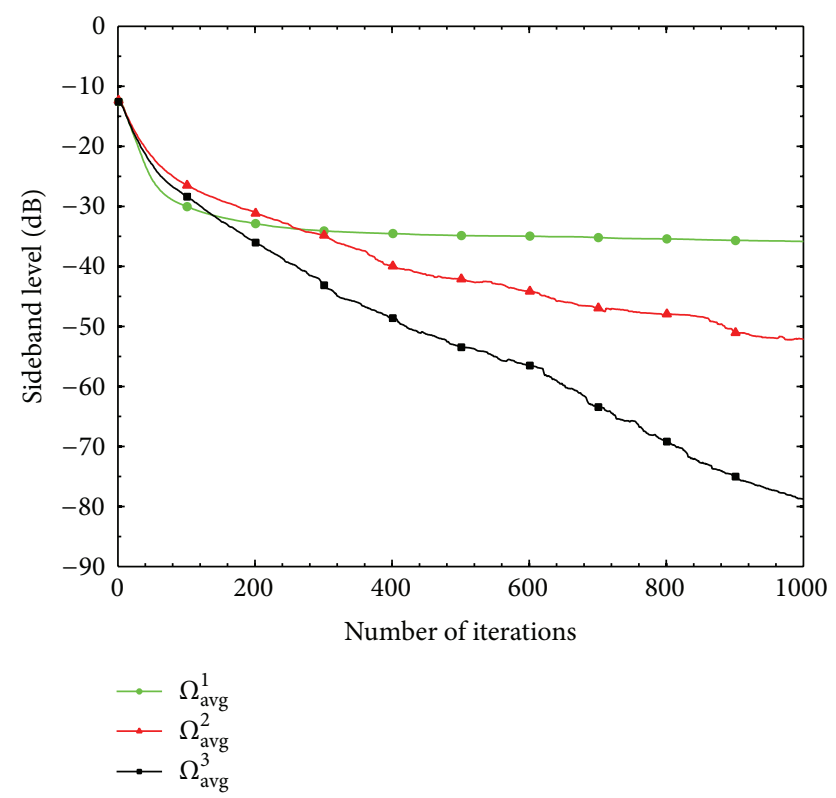

FIGURE 9: Sideband level variation of the three methods for IS $-\Omega^{k}$ represents the maximum sideband level and $k$ represents the method used, where $k=1$ : SBL, $k=2$ : SR, and $k=3$ : SB.

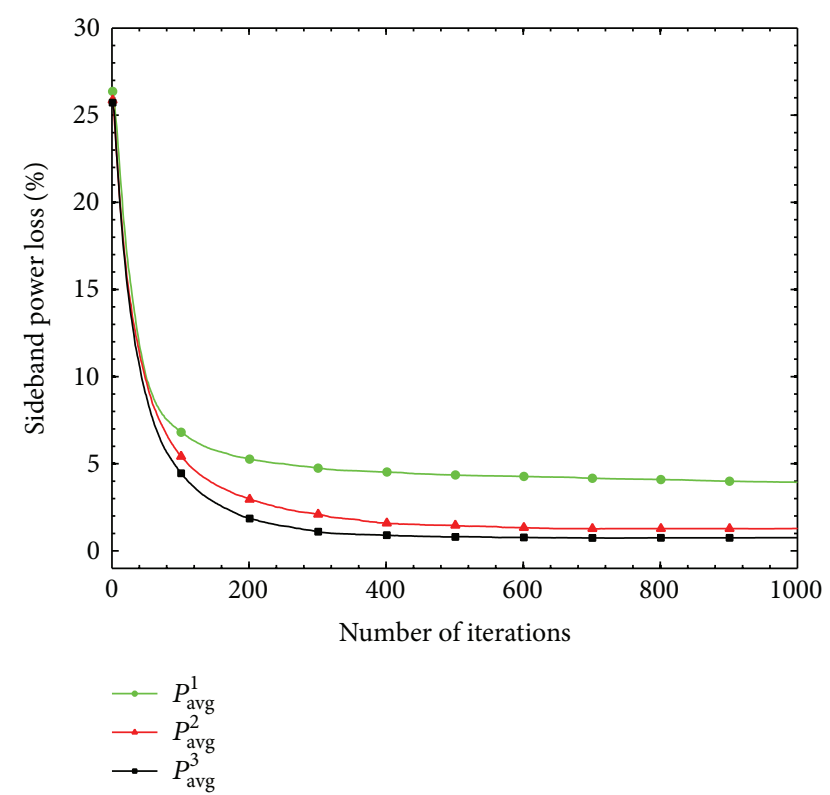

Figure 10: Power loss percentage variation of the three methods for IS $-P^{k}$ represents the power loss percentage and $k$ represents the method used, where $k=1$ : SBL, $k=2: \mathrm{SR}$, and $k=3$ : SB.

The mean time average directivity and the best and the worst results are depicted versus iteration in Figure 11 and tabulated in Table 1, respectively. Regarding the average values, SB method has produced slightly better results than SR method. Since the distance between the array elements is chosen as half-wavelength, efficiency pattern is expected to

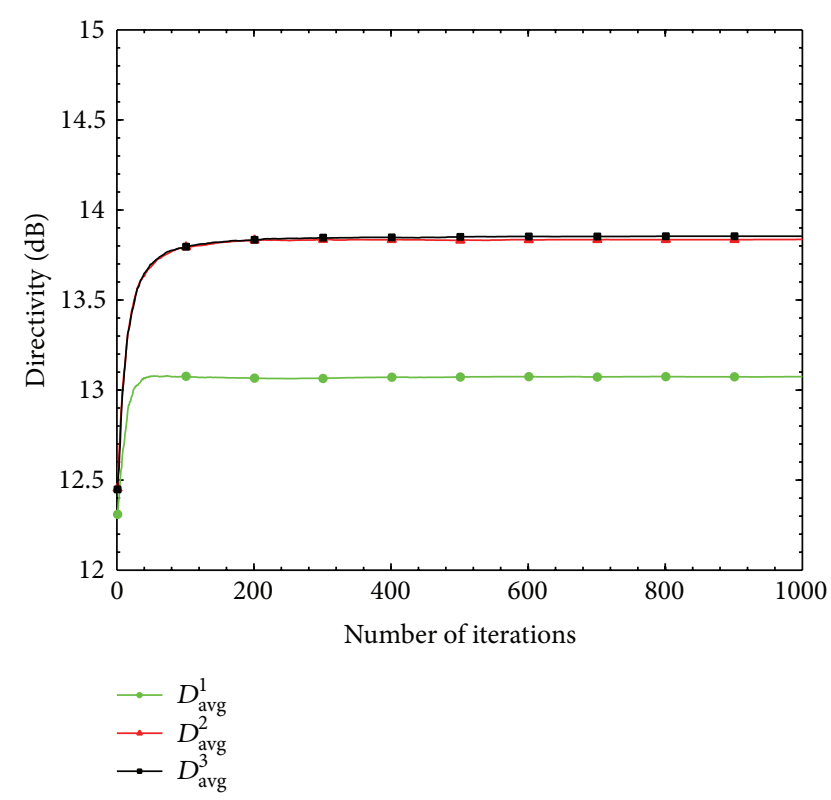

FIGURE 11: Time-average directivity variation of the three methods for IS $-D^{k}$ represents the time-average directivity and $k$ represents the method used, where $k=1$ : SBL, $k=2: \mathrm{SR}$, and $k=3$ : SB.

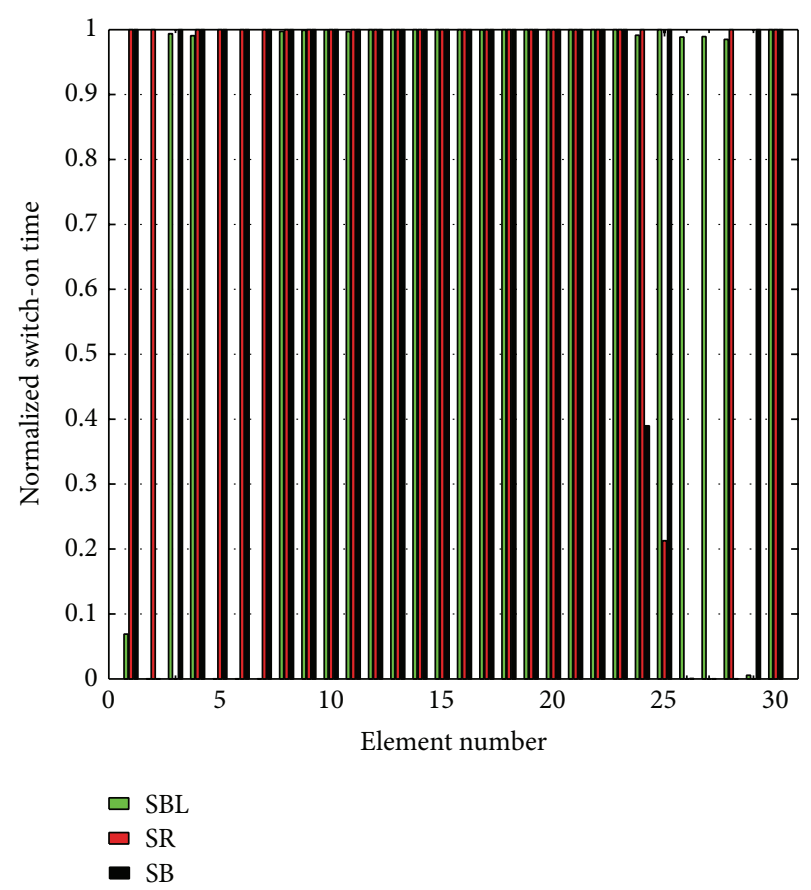

FIGURE 12: Switch-on time durations of the best converged solution for SC.

follow the same pattern with the directivity pattern, so it is not needed to be examined separately. The effect of the methods on solution time is given in Table 2. DE algorithm using SBL method has completed 1000 iterations in $411.85 \mathrm{sec}$; SR and SB methods have completed 1000 iterations in $11.36 \mathrm{sec}$ and $11.02 \mathrm{sec}$, respectively. 


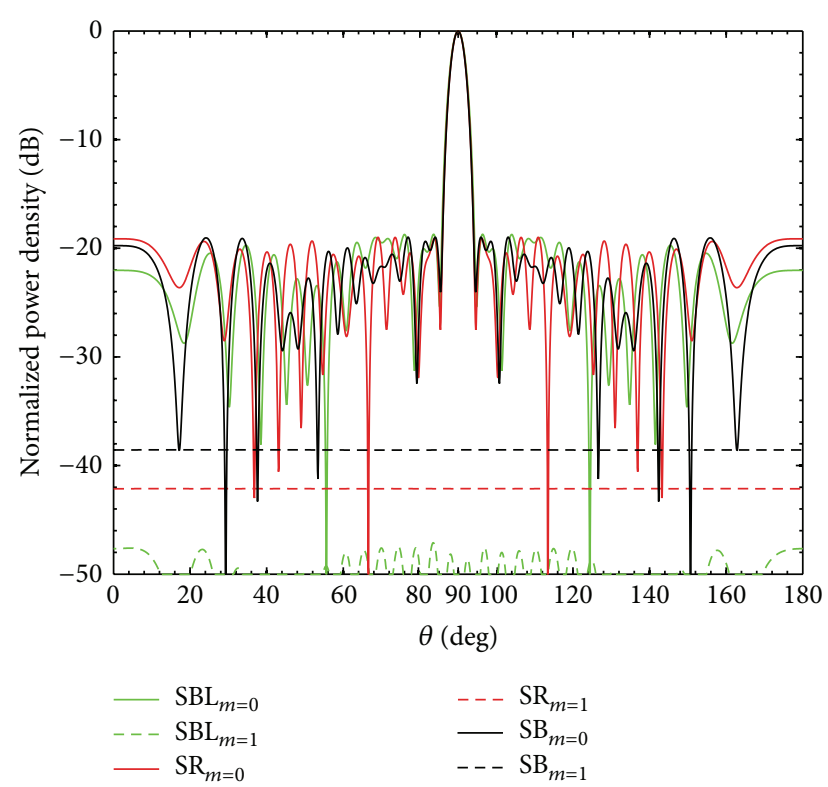

FIGURE 13: Radiation patterns at carrier frequency and at first harmonic related to Figure 12 for SC-the $m$ subscript represents the harmonic number.

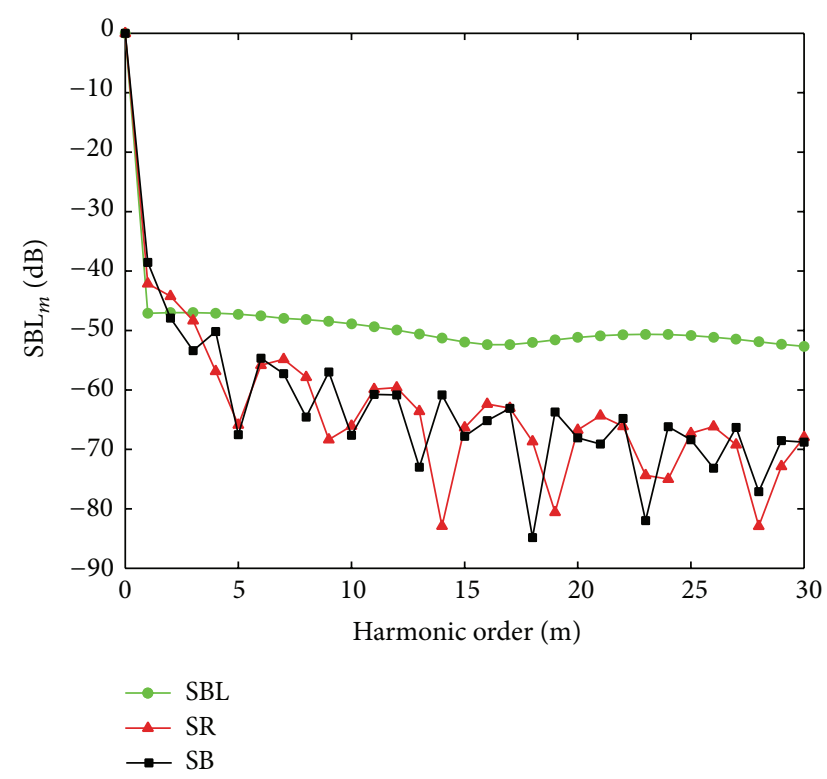

FIgURE 14: The first 30 maximum harmonic levels of best converged solution related to Figure 12 for SC.

TABLE 2: Solution times in seconds.

\begin{tabular}{lccc}
\hline & SBL & SR & SB \\
\hline IS & $411.85 \mathrm{sec}$ & $11.36 \mathrm{sec}$ & $11.02 \mathrm{sec}$ \\
SC & $628.17 \mathrm{sec}$ & $227.43 \mathrm{sec}$ & $226.87 \mathrm{sec}$ \\
JSCIS & $630.42 \mathrm{sec}$ & $228.43 \mathrm{sec}$ & $227.17 \mathrm{sec}$ \\
\hline
\end{tabular}

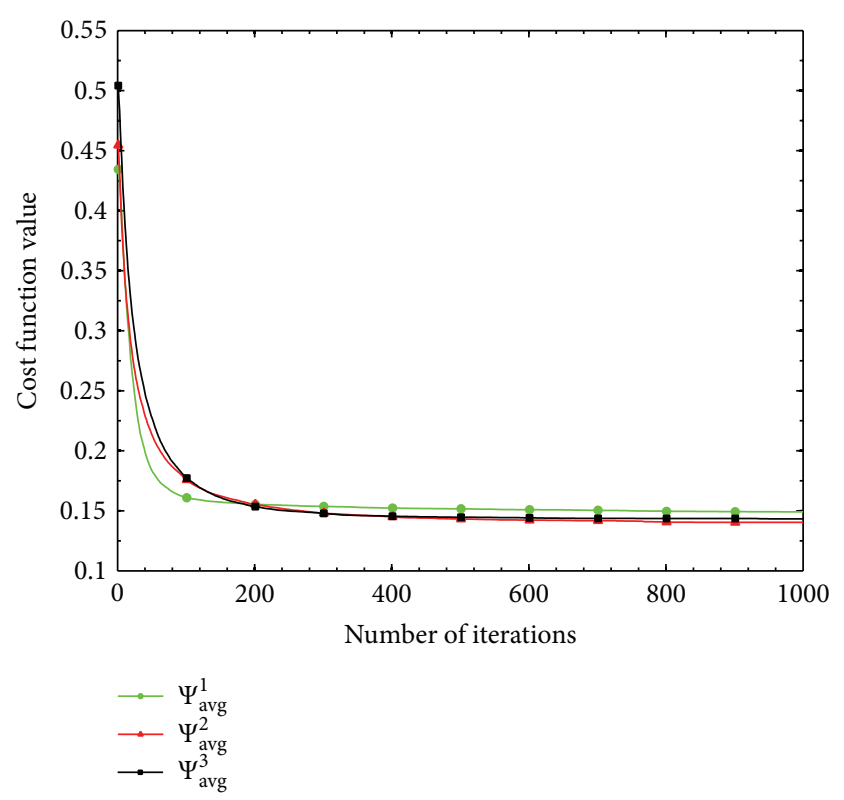

FIGURE 15: The average convergence behaviour of the three methods for SC $-\Psi^{k}$ represents the cost function value and $k$ represents the method used, where $k=1$ : SBL, $k=2$ : SR, and $k=3$ : SB.

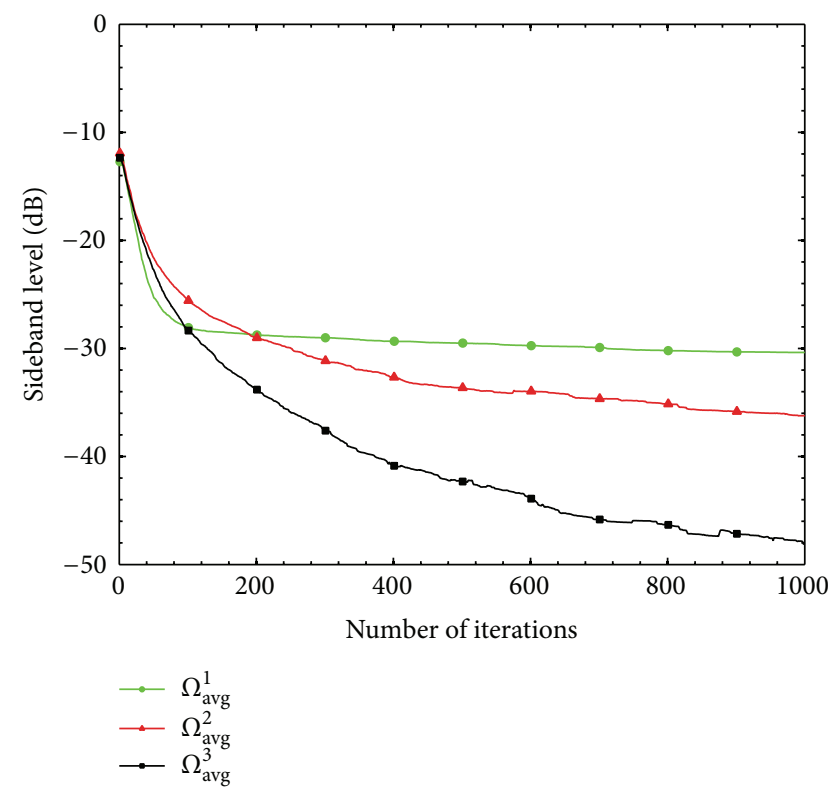

FIGURE 16: Sideband level variation of the three methods for SC $-\Omega^{k}$ represents the maximum sideband level and $k$ represents the method used, where $k=1$ : SBL, $k=2: \mathrm{SR}$, and $k=3$ : SB.

4.2. Sidelobe Cancelling (SC). In this problem, the sideband minimization together with sidelobe level reduction is considered and the cost function given by

$$
\Psi^{k}=\Xi^{k}+\Lambda^{k}
$$

is tried to be minimized by VAS scheme as in the previous problem. In (10), unlike (9), the $\Xi$ term represents the corresponding sidelobe level. For this problem, the optimized 
TABLE 3: Calculated benchmark parameters of DE algorithm outputs for SC.

\begin{tabular}{lccccccccc}
\hline & \multicolumn{3}{c}{ SBL } & \multicolumn{3}{c}{ SC } & & \multicolumn{2}{c}{ SB } \\
& Best & Worst & Mean & Best & Worst & Mean & Best & Worst & Mean \\
\hline$\Psi$ & 0.136 & 0.184 & 0.149 & 0.119 & 0.166 & 0.140 & 0.124 & 0.189 \\
$\Omega(\mathrm{dB})$ & -30.593 & -27.949 & -30.375 & -42.125 & -24.251 & -36.233 & -38.552 & -32.861 & -48.142 \\
$P(\%)$ & 6.593 & 7.570 & 6.044 & 0.665 & 4.987 & 1.923 & 0.939 & 1.808 & 1.070 \\
$D(\mathrm{~dB})$ & 13.797 & 13.537 & 13.680 & 14.017 & 13.886 & 13.950 & 14.047 & 14.187 & 14.031 \\
\hline
\end{tabular}

$\Psi$ represents the cost function value, $\Omega$ represents the maximum sideband level, $P$ represents the power loss percentage, and $D$ represents the time-average directivity.

TABLE 4: Calculated benchmark parameters of DE algorithm outputs for JSCIS.

\begin{tabular}{|c|c|c|c|c|c|c|c|c|c|}
\hline \multicolumn{10}{|c|}{ JSCIS } \\
\hline & \multicolumn{3}{|c|}{ SBL } & \multicolumn{3}{|c|}{ SR } & \multicolumn{3}{|c|}{ SB } \\
\hline & Best & Worst & Mean & Best & Worst & Mean & Best & Worst & Mean \\
\hline$\Psi$ & 0.133 & 0.210 & 0.160 & 0.132 & 0.201 & 0.159 & 0.133 & 0.220 & 0.165 \\
\hline$\Omega(\mathrm{dB})$ & -33.506 & -28.911 & -28.051 & -29.320 & -20.694 & -27.721 & -35.940 & -28.618 & -30.155 \\
\hline$P(\%)$ & 3.929 & 5.976 & 6.814 & 2.926 & 7.796 & 4.055 & 1.317 & 3.224 & 3.094 \\
\hline$D(\mathrm{~dB})$ & 13.907 & 13.565 & 13.695 & 13.821 & 13.836 & 13.922 & 14.052 & 13.533 & 13.962 \\
\hline
\end{tabular}

$\Psi$ represents the cost function value, $\Omega$ represents the maximum sideband level, $P$ represents the power loss percentage, and $D$ represents the time-average directivity.

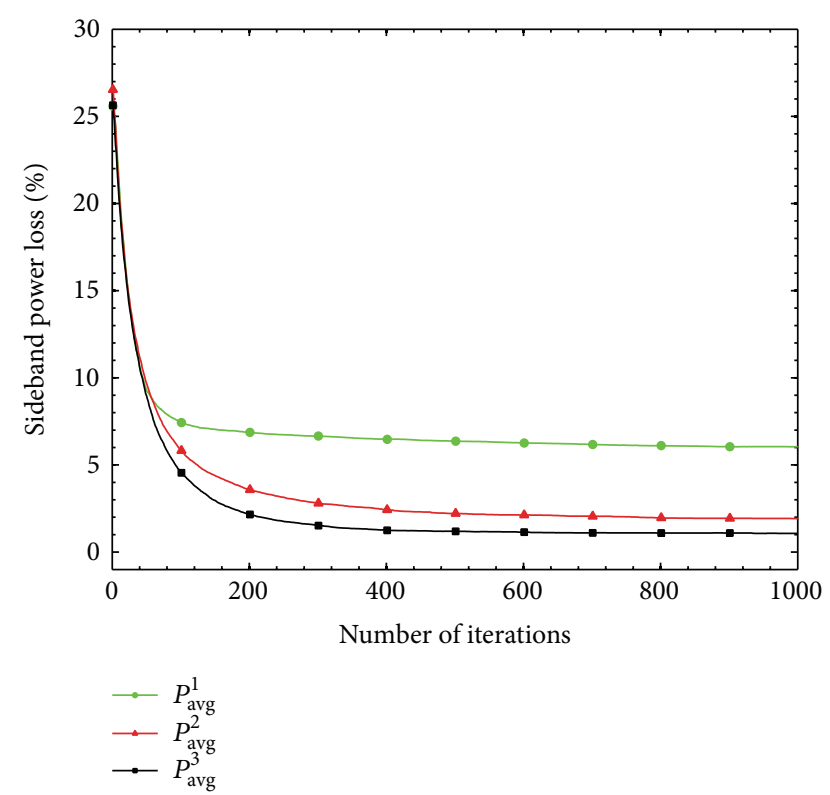

FIGURE 17: Power loss percentage variation of the three methods for $\mathrm{SC}-P^{k}$ represents the power loss percentage and $k$ represents the method used where $k=1$ : SBL, $k=2: \mathrm{SR}$, and $k=3$ : SB.

switch-on durations for the best results amongst 100 experiments are given in Figure 12 and related radiation diagrams are presented in Figure 13. As it can be seen from Figure 13, for all three methods the sidelobe level is taken under $-19 \mathrm{~dB}$ and sideband level is pulled below $-38 \mathrm{~dB}$. Specifically, the first 30 harmonic levels for the solution which has the best cost function value are given in Figure 14. As it can be understood from Figure 14, for the best solution in terms of

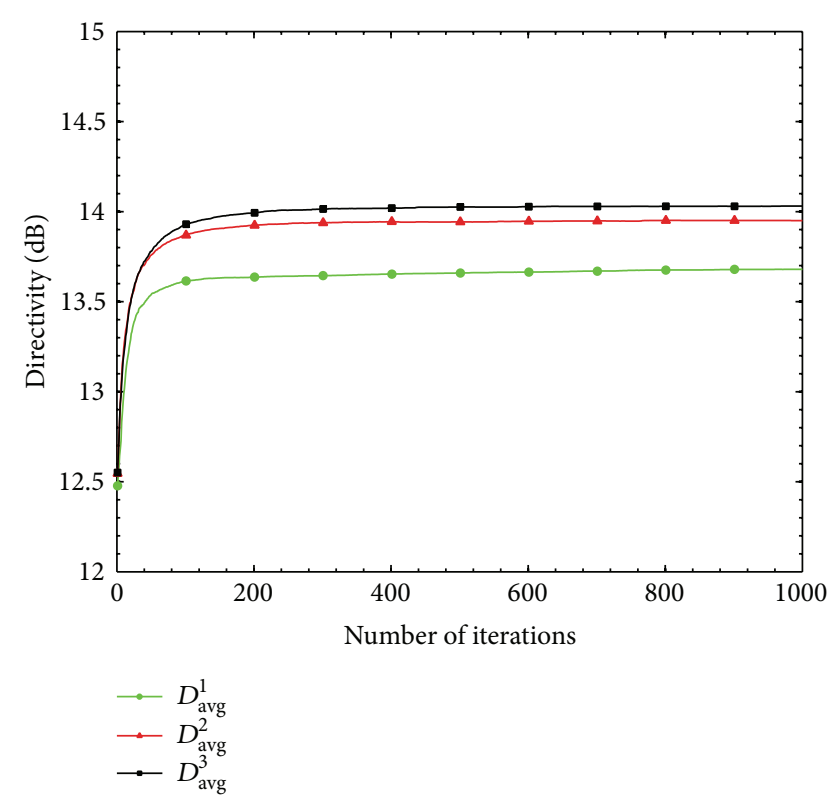

FIGURE 18: Time-average directivity variation of the three methods for $\mathrm{SC}-D^{k}$ represents the time-average directivity and $k$ represents the method used, where $k=1: \mathrm{SBL}, k=2: \mathrm{SR}$, and $k=3$ : SB.

cost function value, the highest sideband level is produced by SB technique, and it is followed by SR and SBL, respectively. Additionally, while the other two methods tend to reduce the higher order sideband levels, SBL technique keeps this level in $-50 \mathrm{~dB}$ neighbourhood. For sidelobe cancelling problem, the calculated best, worst, and average solutions are given in Table 3 and the mean cost function value versus iteration graph is given in Figure 15. For this problem, it can be said 


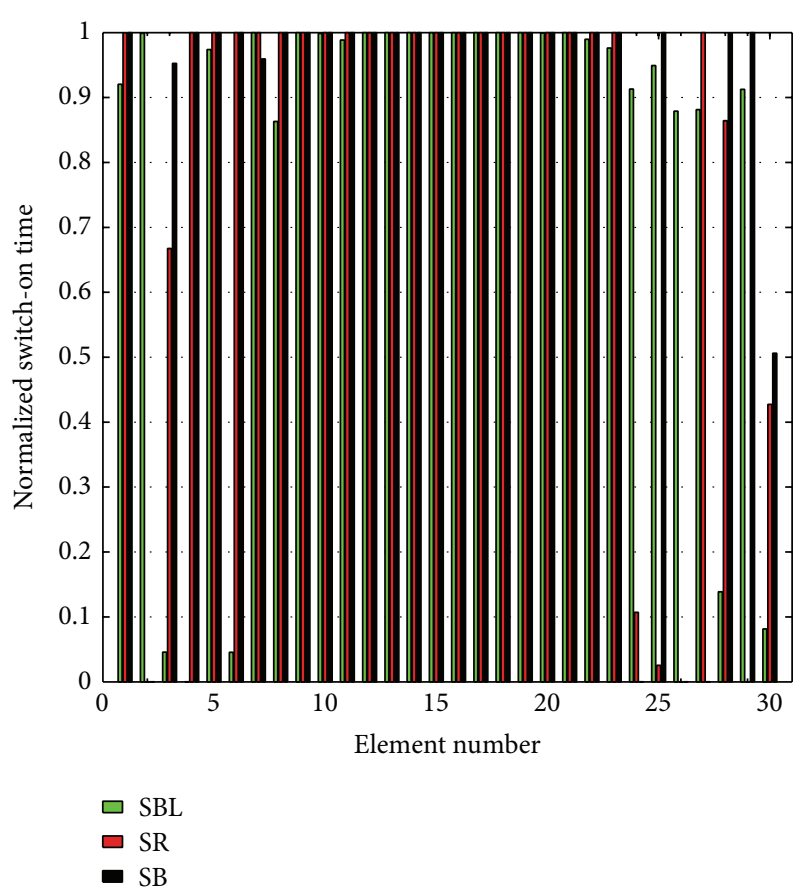

FIGURE 19: Switch-on time durations of the best converged solution for JSCIS.

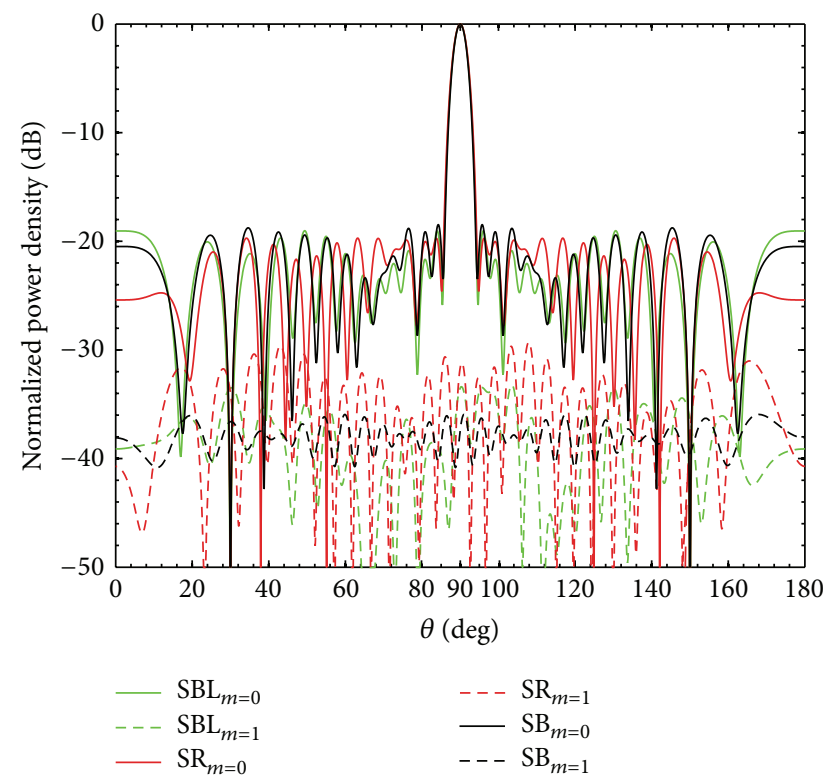

FIGURE 20: Radiation patterns at carrier frequency and at first harmonic related to Figure 19 for JSCIS.

that the convergence of SR method is better than the other two methods in terms of the average values. Considering the average curves, while SR and SB methods decrease in a similar fashion, SBL technique tends to converge fast in early iterations; however, as the number of iterations increases it falls behind the other two methods as in the previous problem.

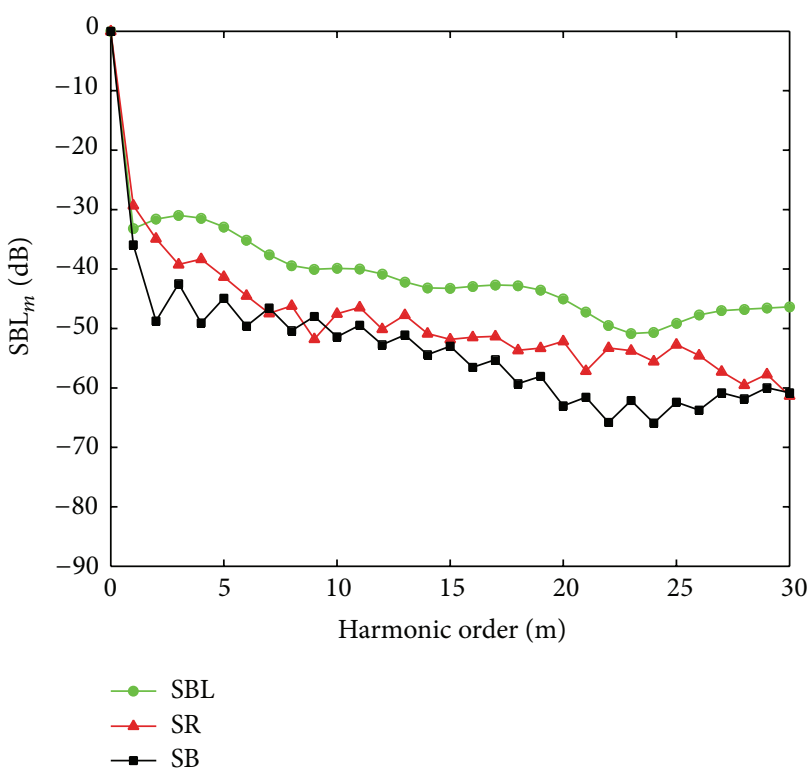

FIGURE 21: The first 30 maximum harmonic levels of best converged solution related to Figure 19 for JSCIS.

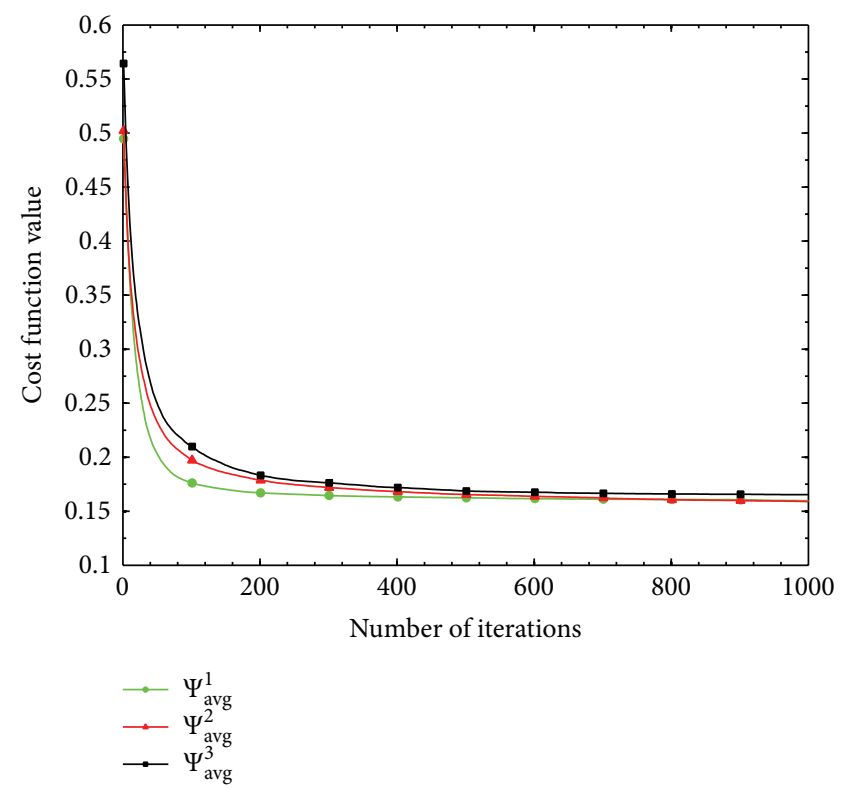

FIGURE 22: The average convergence behaviour of the three methods for JSCIS $-\Psi^{k}$ represents the cost function value and $k$ represents the method used, where $k=1: \mathrm{SBL}, k=2$ : SR, and $k=3$ : SB.

The mean sideband level versus iteration graph is given in Figure 16. Referring to Table 3, SBL and SB methods produce lower values compared to the two other techniques for the best and the worst solutions, respectively. As it can be seen from Figure 16, although SBL method outperforms the other two methods when the best case is considered, SB technique produces better results compared to its rivals for the average case. Additionally, when the tangents of the average curves are taken into account, the sideband suppression behaviour of 
SB method is better than SR technique which behaves better than SBL.

Since SR technique is the process of direct reduction of power, the investigation of the change of power loss as in the first problem would be beneficial. The variation of mean sideband power loss percentage with respect to iteration graph is given in Figure 17. As it can be read from Figure 17, although all three methods succeed in keeping the power loss below \% 1 of total radiated power, the best reduction is realized by SR method.

The mean time average directivity versus iteration graph is given in Figure 18. When Figure 18 is analyzed it is seen that while $\mathrm{SB}$ method reaches an average value of above $14 \mathrm{~dB}$, the SR and SBL methods reach the values around $13.95 \mathrm{~dB}$ and $13.6 \mathrm{~dB}$, respectively. For this problem, as given in Table 2, the solution times obtained by evaluating 1000 iterations for the DE implementations of the methods are calculated as $628.17 \mathrm{sec}, 227.43 \mathrm{sec}$, and $226.87 \mathrm{sec}$, for SBL, SR, and SB methods, respectively.

4.3. Joint Sidelobe Cancelling and Interference Suppression (JSCIS). The last problem examined is the combination of the previous two examples. For this problem, suppressing the sidelobe level and 30-degree elevation angle as in previous problems while attempting to reduce the sideband level with VAS switching scheme is considered. The approach to solve this problem is to reduce the cost function defined by

$$
\Psi^{k}=\Upsilon^{k}+\Xi^{k}+\Lambda^{k}
$$

As in previous problems, the switch-on times for the best solution are given in Figure 19. The radiation patterns and maximum sideband levels of first 30 harmonics related to this switching scheme are reported in Figures 20 and 21, respectively. Additionally, like previous problems, all calculated parameters for JSCIS are given in Table 4.

As it can be seen from Figure 20, the interference suppression is realized and all sidelobes are kept below $-19 \mathrm{~dB}$ while the sideband levels are pulled under $-29 \mathrm{~dB}$. Specifically, if Figure 21 is analyzed, it is found that the highest sideband level obtained for the best solution in 1000 iterations is produced by SR method while the lowest is produced by SB method. If the effect of the methods on the convergence is considered, the mean cost function value versus iteration graph which is given in Figure 22 may be examined. Referring to Table 4, while for the best solution all three methods converge to a common neighbourhood, for the worst solution the convergence ranking from worst to best emerges as $\mathrm{SB}$, SBL, and SR and according to Figure 22, on average, while SBL and SR methods achieve a similar convergence value, SB method has been left behind compared to the other two methods in terms of convergence.

Since the methods are used in the sideband suppression process, examining the sideband level-iteration graph (Figure 23) is beneficial as is done in previous problems. As it can be understood from Figure 23, the best sideband levels are produced from worse to better by SR, SBL, and $\mathrm{SB}$ methods. If the variation of sideband power is examined (Figure 24), while SB method is reducing the sideband power

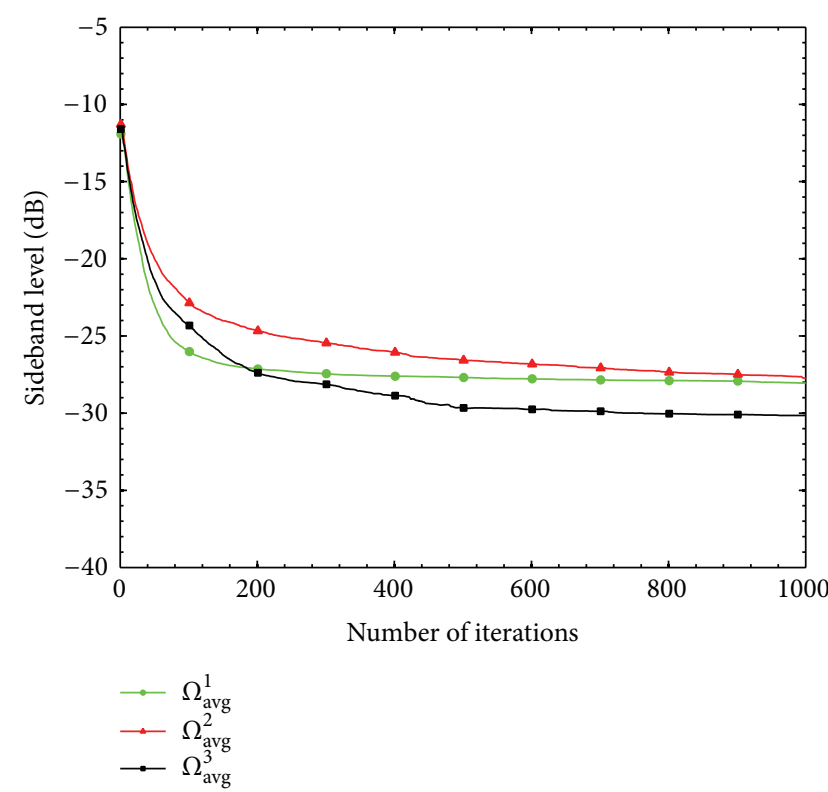

FIGURE 23: Sideband level variation of the three methods for JSCIS$\Omega^{k}$ represents the maximum sideband level and $k$ represents the method used, where $k=1$ : SBL, $k=2$ : SR, and $k=3$ : SB.

to the level of $\% 3$, this value is around $\% 4$ and $\% 6.8$ for SR and SBL methods, respectively. Additionally, it must be noted that while it is expected that SR method which directly reduces sideband radiation power shows a tendency for rapid decrease compared to SBL and SB techniques, it is in fact found that $\mathrm{SB}$ method has reduced the sideband power more rapidly than the other two methods. Moreover, according to Figure 24 the best values produced by SR method are close to that of average values produced by SB method and the best value of $\mathrm{SB}$ method is about $\% 1$ lower than the average value.

Finally, if the variation of time average directivity is examined (Figure 25), on average, SBL method has fallen behind compared to SB and SR. Also, as reported in Table 2, the solution times upon 1000 iterations for this problem are calculated as $630.42 \mathrm{sec}$ for SBL, $228.43 \mathrm{sec}$ for SR, and $227.17 \mathrm{sec}$ for SB.

\section{Discussion}

5.1. Mathematical and Computational Aspects. For timemodulated array problems in which sidebands are considered as a loss, maximum sideband level and total radiated power at sidebands are the two most important parameters. Maximum sideband level is important for the prevention of unwanted communications at the sidebands. So, if the sidebands will not be used for a useful purpose they must be suppressed. As the results obtained from the three methods for the three problems are considered, the newly proposed SB method is found to be more successful than the other two methods in sideband suppression. The second parameter, total radiated sideband power, may be regarded as a loss since it is dissipated in unused sidebands and it must be minimized so as to increase array efficiency. It is expected that SR method would 


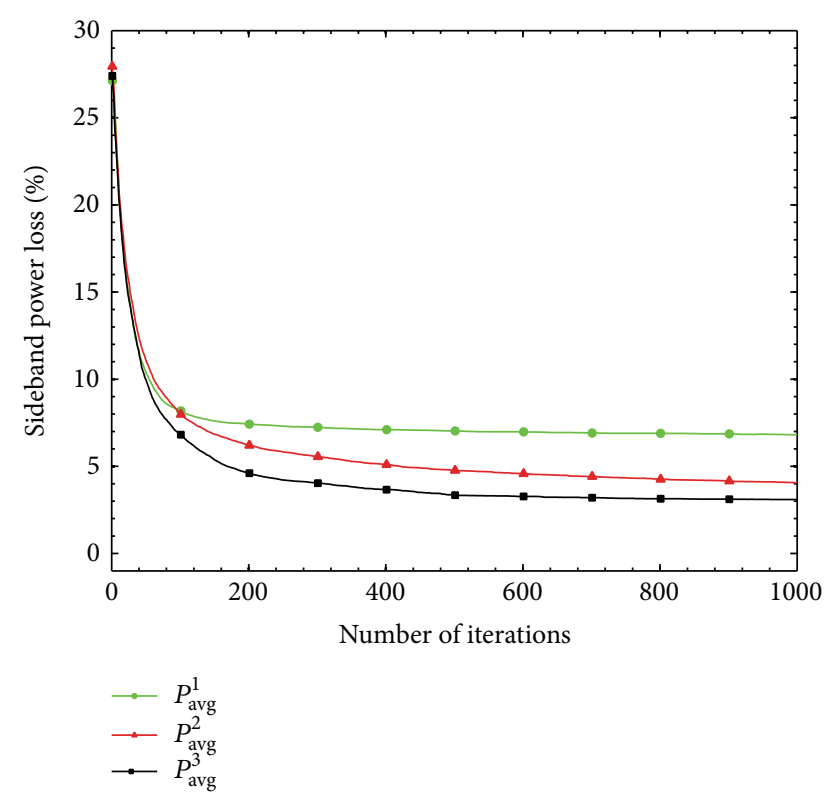

FIGURE 24: Power loss percentage variation of the three methods for JSCIS $-P^{k}$ represents the power loss percentage and $k$ represents the method used, where $k=1$ : SBL, $k=2:$ SR, and $k=3$ : SB.

perform better in reducing sideband power loss since this method directly operates on sideband power. Nevertheless, it is found that SB method outperforms the other two methods for all problems. So, in this respect, at least for the problems taken into account the usage of SB method will be an efficient solution for the sideband level and sideband power suppression. Generally, in time modulation problems, a high directivity accompanied with a low power loss and a low sideband level is required. SB method has been found to maintain better directivity values for three problems. Although efficiency is not directly considered in this paper, the efficiency pattern will be equivalent to directivity pattern since the distance between the array elements is halfwavelength. In this case, SB method will give more successful results in increasing efficiency.

From the perspective of solution time, SBL method requires approximately three times more time than the other two methods, although an azimuth symmetry is present. This ratio is expected to increase incredibly for planar and volumetric arrays. This situation shows that SR and SB methods are more useful than SBL method regarding solution time. SR and SB methods offer solution times close to each other. At this point it must be noticed that in SR method whenever the arrays having half-wavelength interelement distances are used, the second summation in (7) converges to zero and may be omitted, and this situation (as is used in this work) shortens the solution time. Therefore, regarding solution times SB method has advantage over the other two methods.

5.2. Practical Aspects. In the evaluation of the methods, the area of applicability is also important and applicability status of these three methods in terms of geometric structure
TABLE 5: Current applicability status of the three methods.

\begin{tabular}{lcccccc}
\hline & \multicolumn{2}{c}{ SBL } & \multicolumn{2}{c}{ SR } & \multicolumn{2}{c}{ SB } \\
& VAS & PS & VAS & PS & VAS & PS \\
\hline Linear & $\checkmark$ & $\checkmark$ & $\checkmark$ & $\checkmark$ & $\checkmark$ & $x$ \\
Planar & $\checkmark$ & $\checkmark$ & $\checkmark$ & $\checkmark$ & $\checkmark$ & $x$ \\
Volumetric & $\checkmark$ & $\checkmark$ & $x$ & $x$ & $x$ & $x$ \\
\hline
\end{tabular}

Check and cross signs represent the applicability conditions.

and switching type are tabulated in Table 5. SBL method has an algorithmic structure and is independent of array type, but the obtained results show that this method is more ineffective than the other two methods when the others are usable. Since there is not an equation already published for volumetric arrays, SR method is only valid for linear and planar arrays [30]. The normalization term of (8) is written only for linear and planar arrays, so SB method can be used also only for linear and planar arrays. Moreover, SR method is generalized for VAS and pulse shift switching [31], but this is not valid for SB already. Although SB method may be used for pulse shifting scheme, the control of the method over pulse shifting scheme is found to be weak since the inequality does not contain a term including pulse beginning times and is marked with cross sign in Table 5. Already, this seems to be the only handicap of the SB method.

The results given in the analysis section (Section 4) have been simulated theoretically and calculated without taking into account any physical condition. Hence, any constraint has not been applied during all these optimization procedures. This situation has led to some switching times that are very close to null or unitary values which may be hard to realize in practice. A null switching time means that the element is always turned off and a unitary switching time means that the element is always turned on. In practice, for both situations the time modulation concept disappears and for real applications minimum and maximum switching duration constraints should be added to the optimization process. Also, switching times may be optimized in a discrete space instead of a continuous space, although this may reduce the sensitivity.

Additionally, the given solutions are the results directly obtained from theoretical calculations without any constraints and actually $-130 \mathrm{~dB}$ or $-140 \mathrm{~dB}$ values as observed in the IS problem are very hard to achieve or even impossible in practice. In fact, suppressing a radiated quantity below $-50 \mathrm{~dB}$ makes no sense in practice because it is very difficult to realize or even unmeasurable. From this perspective, inserting an additional minimum sideband level constraint to the optimization process would be beneficial in order to make the design more realizable. Moreover, suppressing the sidebands forces the switching times to tend to null or unitary values and the number of time modulated elements to be reduced in number. Hence, this situation reduces the time modulation effect on dynamic excitations and the degrees of freedom gained by time parameter in array design. Therefore, in practice, a restriction of the SBL in accordance with a proper constraint is suggested. 


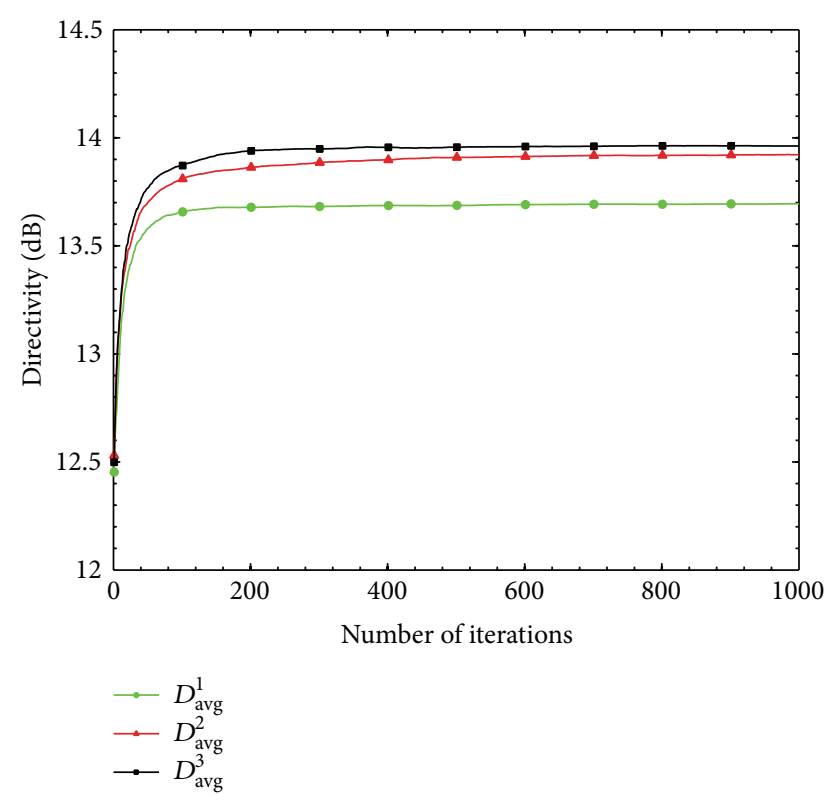

FIGURE 25: Time-average directivity variation of three methods for JSCIS $-D^{k}$ represents the time-average directivity and $k$ represents the method used where $k=1$ : SBL, $k=2$ : SR, $k=3$ : SB.

\section{Conclusion}

In this work, two already existing sideband determination and control techniques (i.e., SBL and SR) and a recently proposed one (i.e., SB) are investigated and analyzed in a comparative fashion over three different problems named interference suppression, sidelobe cancelling, and joint interference suppression and sidelobe cancelling. The problems are constructed on a linear array with half-wavelength interelement spacing and the techniques are analyzed in terms of sideband level, sideband power, time average directivity, and solution time. The obtained results show that the recently proposed SB technique exhibits better results in terms of sideband level, sideband power, and directivity than the other two compared existing methods theoretically. As a result, it can be said that in accordance with the data obtained, SB method providing shorter computation time is more preferable than the compared methods in terms of the analysis benchmarks. Additionally, some comments and suggestions are given for the optimization process in terms of practical aspects.

\section{Conflict of Interests}

The authors declare that there is no conflict of interests regarding the publication of this paper.

\section{References}

[1] H. E. Shanks and R. W. Bickmore, "Four-dimensional electromagnetic radiators," Canadian Journal of Physics, vol. 37, no. 3 , pp. 263-275, 1959.
[2] W. H. Kummer, A. T. Villeneuve, T. S. Fong, and F. G. Terrio, "Ultralow sidelobes from time-modulated arrays," IEEE Transactions on Antennas and Propagation, vol. 11, no. 6, pp. 633-639, 1963.

[3] R. W. Bickmore, "Time versus space in antenna theory," in Microwave Scanning Antennas, R. C. Hansen, Ed., vol. 3, Academic Press, New York, NY, USA, 1966.

[4] S. Yang, Y. B. Gan, and A. Qing, "Sideband suppression in timemodulated linear arrays by the differential evolution algorithm," IEEE Antennas and Wireless Propagation Letters, vol. 1, no. 1, pp. 173-175, 2002.

[5] E. Aksoy and E. Afacan, "Thinned nonuniform amplitude time-modulated linear arrays," IEEE Antennas and Wireless Propagation Letters, vol. 9, pp. 514-517, 2010.

[6] L. Poli, P. Rocca, L. Manica, and A. Massa, "Handling sideband radiations in time-modulated arrays through particle swarm optimization," IEEE Transactions on Antennas and Propagation, vol. 58, no. 4, pp. 1408-1411, 2010.

[7] J. Fondevila, J. C. Brégains, F. Ares, and E. Moreno, "Optimizing uniformly excited linear arrays through time modulation," IEEE Antennas and Wireless Propagation Letters, vol. 3, no. 1, pp. 298301, 2004.

[8] G. Li, S. Yang, M. Huang, and Z. Nie, "Sidelobe suppression in time modulated linear arrays with unequal element spacing," Journal of Electromagnetic Waves and Applications, vol. 24, no. 5-6, pp. 775-783, 2010.

[9] S. Yang, Y. B. Gan, and P. K. Tan, "Comparative study of low sidelobe time modulated linear arrays with different time schemes," Journal of Electromagnetic Waves and Applications, vol. 18, no. 11, pp. 1443-1458, 2004.

[10] L. Poli, P. Rocca, L. Manica, and A. Massa, "Pattern synthesis in time-modulated linear arrays through pulse shifting," IET Microwaves, Antennas and Propagation, vol. 4, no. 9, pp. 1157$1164,2010$.

[11] S. Yang, Y. B. Gan, A. Qing, and P. K. Tan, "Design of a uniform amplitude time modulated linear array with optimized time sequences," IEEE Transactions on Antennas and Propagation, vol. 53, no. 7, pp. 2337-2339, 2005.

[12] E. Aksoy and E. Afacan, "Sideband level suppression improvement via splitting pulses in time modulated arrays under static fundamental radiation," in Proceedings of the Progress in Electromagnetics Research Symposium (PIERS '11), pp. 364-367, Suzhou, China, September 2011.

[13] Q. Zhu, S. Yang, L. Zheng, and Z. Nie, "Design of a low sidelobe time modulated linear array with uniform amplitude and subsectional optimized time steps," IEEE Transactions on Antennas and Propagation, vol. 60, no. 9, pp. 4436-4439, 2012.

[14] Y. Tong and A. Tennant, "Sideband level suppression in timemodulated linear arrays using modified switching sequences and fixed bandwidth elements," Electronics Letters, vol. 48, no. 1, pp. 10-11, 2012.

[15] L. Poli, P. Rocca, G. Oliveri, and A. Massa, "Adaptive nulling in time-modulated linear arrays with minimum power losses," IET Microwaves, Antennas and Propagation, vol. 5, no. 2, pp. 157-166, 2011.

[16] E. Aksoy and E. Afacan, "Control of the sideband level and pattern null in time modulated linear arrays," in Proceedings of the IEEE 19th Signal Processing and Communications Applications Conference (SIU '11), pp. 351-354, Antalya, Turkey, April 2011.

[17] P. Rocca, L. Poli, G. Oliveri, and A. Massa, "Adaptive nulling in time-varying scenarios through time-modulated linear arrays," 
IEEE Antennas and Wireless Propagation Letters, vol. 11, pp. 101104, 2012.

[18] Y. Tong and A. Tennant, "A two-channel time modulated linear array with adaptive beamforming," IEEE Transactions on Antennas and Propagation, vol. 60, no. 1, pp. 141-147, 2012.

[19] A. Tennant and B. Chambers, "A two-element time-modulated array with direction-finding properties," IEEE Antennas and Wireless Propagation Letters, vol. 6, pp. 64-65, 2007.

[20] A. Tennant, "Experimental two-element time-modulated direction finding array," IEEE Transactions on Antennas and Propagation, vol. 58, no. 3, pp. 986-988, 2010.

[21] G. Li, S. Yang, Y. Chen, and Z. Nie, "A novel electronic beam steering technique in time modulated antenna arrays," Progress in Electromagnetics Research, vol. 97, pp. 391-405, 2009.

[22] G. Li, S. Yang, and Z. Nie, "Direction of arrival estimation in time modulated linear arrays with unidirectional phase center motion," IEEE Transactions on Antennas and Propagation, vol. 58, no. 4, pp. 1105-1111, 2010.

[23] T. Hong, M.-Z. Song, and Y. Liu, "RF directional modulation technique using a switched antenna array for communication and direction-finding applications," Progress in Electromagnetics Research, vol. 120, pp. 195-213, 2011.

[24] Q. Zhu, S. Yang, R. Yao, and Z. Nie, "Gain improvement in timemodulated linear arrays using SPDT switches," IEEE Antennas and Wireless Propagation Letters, vol. 11, pp. 994-997, 2012.

[25] Y. Wang and A. Tennant, "Time-modulated reflector array," Electronics Letters, vol. 48, no. 16, pp. 972-974, 2012.

[26] P. Rocca, L. Poli, and A. Massa, "Instantaneous directivity optimization in time-modulated array receivers," IET Microwaves, Antennas \& Propagation, vol. 6, no. 14, pp. 1590-1597, 2012.

[27] E. T. Bekele, L. Poli, P. Rocca, M. D’Urso, and A. Massa, "Pulse-shaping strategy for time modulated arrays-analysis and design," IEEE Transactions on Antennas and Propagation, vol. 61, no. 7, pp. 3525-3537, 2013.

[28] J. C. Brégains, J. Fondevila-Gómez, G. Franceschetti, and F. Ares, "Signal radiation and power losses of time-modulated arrays," IEEE Transactions on Antennas and Propagation, vol. 56, no. 6, pp. 1799-1804, 2008.

[29] L. Poli, P. Rocca, L. Manica, and A. Massa, “Time modulated planar arrays-analysis and optimisation of the sideband radiations," IET Microwaves, Antennas and Propagation, vol. 4, no. 9, pp. 1165-1171, 2010.

[30] E. Aksoy and E. Afacan, "Generalized representation of sideband radiation power calculation in arbitrarily distributed time-modulated planar and linear arrays," in Proceedings of the Progress in Electromagnetics Research Symposium (PIERS '11), pp. 368-371, Suzhou, China, September 2011.

[31] E. Aksoy and E. Afacan, "Calculation of sideband power radiation in time-modulated arrays with asymmetrically positioned pulses," IEEE Antennas and Wireless Propagation Letters, vol. 11, pp. 133-136, 2012.

[32] E. Aksoy and E. Afacan, "An inequality for the calculation of relative maximum sideband level in time-modulated linear and planar arrays," IEEE Transactions on Antennas and Propagation, no. 99, 1 page, 2014.

[33] E. Aksoy, "Design of asymmetrically switched linear Chebyshev array with minimum harmonic power loss," in Proceedings of the 9th International Conference on Electronics, Computer and Computation (ICECCO '12), pp. 112-115, Ankara, Turkey, November 2012. 

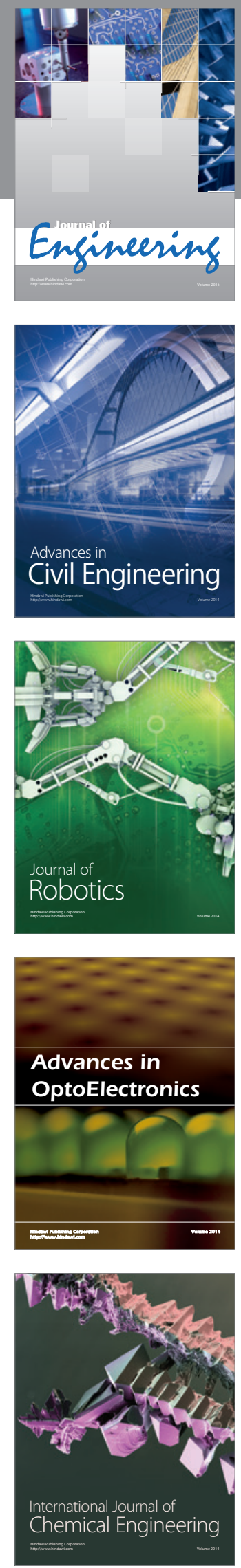

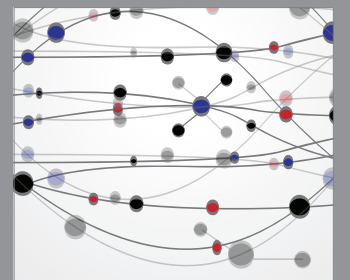

The Scientific World Journal
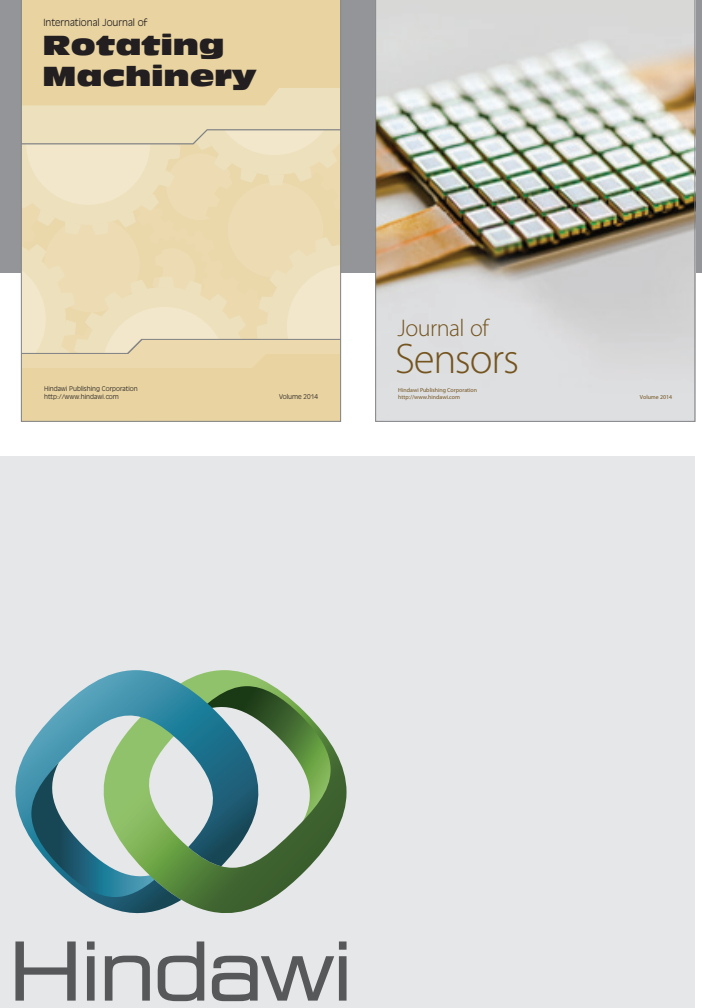

Submit your manuscripts at http://www.hindawi.com
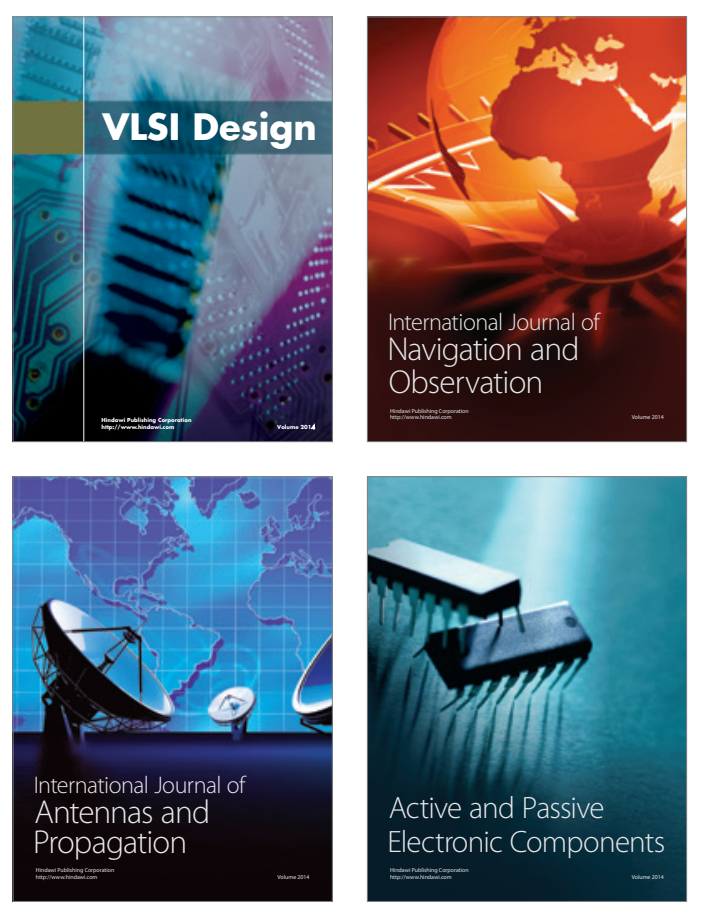
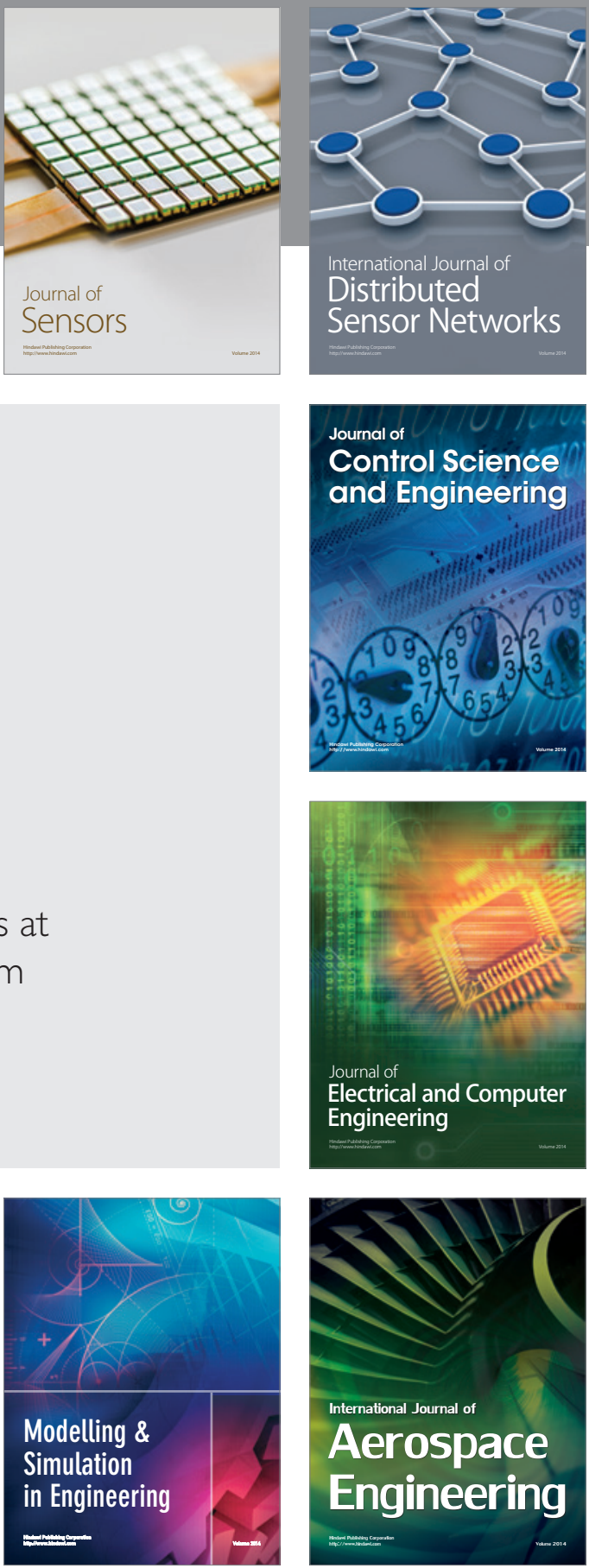

Journal of

Control Science

and Engineering
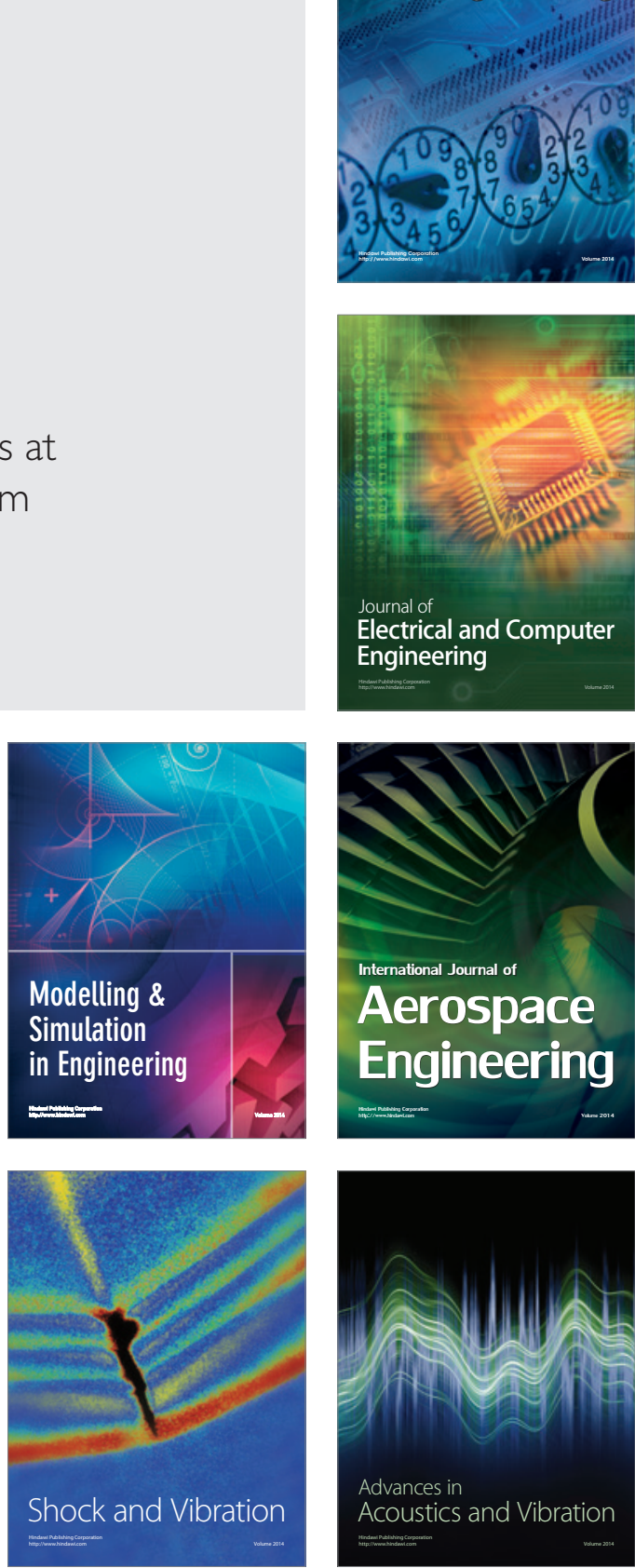